\title{
A Computer-Aided Approach for the Discovery of D-Peptides as Inhibitors of SARS-CoV-2 Main Protease
}

\author{
Jorge E. Hernández González ${ }^{1,2}$, Raphael J. Eberle ${ }^{3,4}$, Dieter Willbold ${ }^{3,4,5}$ and \\ Mônika A. Coronado ${ }^{3 *}$ \\ ${ }^{1}$ Multiuser Center for Biomolecular Innovation, IBILCE, Universidade Estadual Paulista (UNESP), São Jose do Rio Preto, Brazil, \\ ${ }^{2}$ Laboratory for Molecular Modeling and Dynamics, Instituto de Biofísica Carlos Chagas Filho, Universidade Federal do Rio de \\ Janeiro, Cidade Universitária Ilha do Fundão, Rio de Janeiro, Brazil, ${ }^{3}$ Institute of Biological Information Processing (IBI-7, \\ Structural Biochemistry), Forschungszentrum Jülich, Jülich, Germany, ${ }^{4}$ Institut für Physikalische Biologie, Heinrich-Heine- \\ Universität Düsseldorf, Universitätsstraße, Düsseldorf, Germany, ${ }^{5} J u S t r u c t:$ Jülich Centre for Structural Biology, \\ Forschungszentrum Jülich, Jülich, Germany
}

\section{OPEN ACCESS}

Edited by: Mattia Falconi, University of Rome Tor Vergata, Italy

Reviewed by: Aditya K. Padhi,

RIKEN Yokohama, Japan K. Venkateswara Swamy, MIT School of Bioengineering Sciences and Research, India

*Correspondence: Mônika A. Coronado m.coronado@fz-juelich.de

Specialty section:

This article was submitted to Biological Modeling and Simulation, a section of the journal

Frontiers in Molecular Biosciences

Received: 16 November 2021 Accepted: 30 December 2021 Published: 24 January 2022

Citation: Hernández González JE, Eberle RJ, Willbold D and Coronado MA (2022) A

Computer-Aided Approach for the Discovery of D-Peptides as Inhibitors of SARS-CoV-2 Main Protease.

Front. Mol. Biosci. 8:816166. doi: 10.3389/fmolb.2021.816166
The SARS-CoV-2 main protease, also known as 3-chymotrypsin-like protease (3CL ${ }^{\text {pro }}$ ), is a cysteine protease responsible for the cleavage of viral polyproteins pp1a and pp1ab, at least, at eleven conserved sites, which leads to the formation of mature nonstructural proteins essential for the replication of the virus. Due to its essential role, numerous studies have been conducted so far, which have confirmed $3 \mathrm{CL}^{\text {pro }}$ as an attractive drug target to combat Covid19 and have reported a vast number of inhibitors and their co-crystal structures. Despite all the ongoing efforts, D-peptides, which possess key advantages over L-peptides as therapeutic agents, have not been explored as potential drug candidates against $3 \mathrm{CL}^{\text {pro }}$. The current work fills this gap by reporting an in silico approach for the discovery of D-peptides capable of inhibiting $3 \mathrm{CL}^{\text {pro }}$ that involves structure-based virtual screening (SBVS) of an in-house library of D-tripeptides and D-tetrapeptides into the protease active site and subsequent rescoring steps, including Molecular Mechanics Generalized-Born Surface Area (MM-GBSA) free energy calculations and molecular dynamics (MD) simulations. In vitro enzymatic assays conducted for the four top-scoring D-tetrapeptides at $20 \mu \mathrm{M}$ showed that all of them caused 55-85\% inhibition of $3 \mathrm{CL}^{\text {pro }}$ activity, thus highlighting the suitability of the devised approach. Overall, our results present a promising computational strategy to identify D-peptides capable of inhibiting $3 \mathrm{CL}{ }^{\text {pro, }}$, with broader application in problems involving protein inhibition.

Keywords: SARS-CoV-2, 3CL ${ }^{\text {pro }}$, D-peptide, virtual screening, molecular dynamics simulation

\section{INTRODUCTION}

Covid-19 is a pandemic disease caused by the novel acute respiratory syndrome coronavirus 2 (SARS-CoV-2). As of December 12th, 2021, over 269 million confirmed Covid-19 cases and 5.3 million related deaths had been reported since the start of the pandemic (World Health Organization, 2021). SARS-CoV-2, together with SARS-CoV and Middle East Respiratory Syndrome (MERS) coronaviruses responsible for two significant outbreaks during the current century, are enveloped and single-stranded RNA viruses (Payne, 2017; Wu F. et al., 2020; Wang et al., 2020). During their replication, coronaviruses encode several accessory proteins and two replicase polyproteins (ppla and pplab) (Marra et al., 2003; Rota et al., 2003; Ziebuhr, 2005; Wu F. et al., 2020; Yan and $\mathrm{Wu}, 2021$ ), which are proteolytically processed by two cysteine proteases, i.e., the papain- 
like protease $\left(\mathrm{PL}^{\mathrm{pro}}\right)$ and the main protease, also called 3chymotrypsin-like protease $\left(3 \mathrm{CL}^{\mathrm{pro}}\right)$. The latter cleaves the ppla and pplb at 11 conserved sites by recognizing the XXXLQAXXX and XXXLQSXXX sequence motifs, thus generating nonstructural proteins (NSPs) essential for the viral replication (Gorbalenya et al., 1989; Hegyi and Ziebuhr, 2002; Kiemer et al., 2004; Yan and Wu, 2021).

The essential role played by SARS-CoV-2 $3 \mathrm{CL}^{\text {pro }}$ during the viral replication has encouraged the search for anti-Covid drugs targeting this protease. Numerous potent orthosteric inhibitors of $3 \mathrm{CL}^{\text {pro }}$, most of them of peptide-based or peptidomimetic nature, have been reported so far (Amin et al., 2021; Chia et al., 2021; Sabbah et al., 2021; Yan and Gao, 2021). These compounds have shown significant inhibitory activity, not only against the protease but also against the viral replication in cell cultures. Meanwhile, the crystal structures of $3 \mathrm{CL}^{\text {pro }}$ in complex with a myriad of inhibitors and compound fragments have been deposited in the Protein Data Bank (PDB) and provide useful structural information for the rational design of new drugs (Mengist et al., 2021). Some of these structures have revealed the existence of allosteric binding sites in the surface of $3 \mathrm{CL}^{\text {pro }}$, which can also be exploited to search for noncompetitive inhibitors (Douangamath et al., 2020; Gunther et al., 2021). More recently, the $3 \mathrm{CL}^{\text {pro }}$ peptidomimetic inhibitor PF-07321332 has shown promising results in phase I clinical trials, thus paving the way toward the discovery of an effective antiviral (Owen et al., 2021). All these results underscore the importance of $3 \mathrm{CL}^{\text {pro }}$ as an attractive drug target to combat Covid-19.

Even though diverse scaffolds of $3 \mathrm{CL}^{\text {pro }}$ inhibitors have been identified, D-peptides remain unexplored. These molecules are made up of D-amino acids, i.e., amino acids whose chiral $\mathrm{Ca}$ atoms have the opposite stereochemical configuration to that observed in the amino acids that commonly form the natural proteins (the L-amino acids). This structural feature endows D-peptides with key advantages over the L-peptides, such as higher stability to proteolysis, improved intestinal absorption upon oral administration, and low or missing immunogenicity. These properties, along with others shared with L-peptides, e.g., lower manufacturing costs and higher binding affinity and specificity for the target receptors in comparison with small molecules, make D-peptides attractive therapeutic agents (Wiesehan and Willbold, 2003; Funke and Willbold, 2009; Sun et al., 2012; Liu et al., 2016; Garton et al., 2018). Remarkably, $\alpha$ helical D-peptides designed in silico were reported to block the binding of the SARS-CoV-2 spike protein receptor-binding domain (RBD) to the human angiotensin-converting enzyme 2 (ACE2), the molecule that mediates the virus internalization into human cells, thus leading to the inhibition of viral infection in vitro (Valiente et al., 2021). The previous results provide an excellent example of the use of D-peptides as promising anti-Covid drug candidates.

Structure-based virtual screening (SBVS) of diverse ligand databases, many of them containing drug repurposing candidates and natural products, has been extensively applied to identify potential $3 \mathrm{CL}^{\text {pro }}$ inhibitors (Wu C. et al., 2020; Chowdhury et al., 2020; Jukic et al., 2020; Meyer-Almes, 2020; Olubiyi et al., 2020; Selvaraj et al., 2020; Federico et al., 2021; Gogoi et al., 2021; Guedes et al., 2021; Kumar et al., 2021; Lokhande et al., 2021; Naik et al., 2021; Rajpoot et al., 2021;
Rehman et al., 2021; Sisakht et al., 2021). In several cases, this approach has led to the successful identification of compounds displaying in vitro inhibitory activity against $3 \mathrm{CL}^{\text {pro }}$ (Ghahremanpour et al., 2020; Gupta et al., 2020; Jin et al., 2020; Li et al., 2020; Alves et al., 2021; Banerjee et al., 2021; Gunther et al., 2021; Guo et al., 2021; Gupta et al., 2021; Hamdy et al., 2021; Pathak et al., 2021; Yang et al., 2021). On the other hand, protein-peptide docking remains far more challenging compared to other small molecules due to the higher flexibility of peptides (Rentzsch and Renard, 2015; Ciemny et al., 2018; Hashemi et al., 2021). Nonetheless, at least one work has reported two L-pentapeptides as potential $3 \mathrm{CL}^{\text {pro }}$ inhibitors by screening a 70,000-peptide library (Porto, 2021), using AutoDock Vina for the docking simulations (Trott and Olson, 2010). Remarkably, AutoDock Vina outperformed other freely-available docking algorithms, such as AutoDock and ZDOCK (Chen and Weng, 2002; Morris et al., 2009), in a benchmark study that presented a pipeline for peptide SBVS (Ansar and Vetrivel, 2019).

Encouraged by the previous findings, this study presents D-peptides as $3 \mathrm{CL}^{\text {pro }}$ inhibitors. The computational workflow employed for D-peptide identification, which will be fully described in Materials and Methods, selects the best binders to the protease active site through SBVS and a series of rescoring steps combining Molecular Mechanics Generalized-Born Surface Area (MM-GBSA) free energy calculations (Gohlke et al., 2003; Gohlke and Case, 2004; Miller et al., 2012) and molecular dynamics (MD) simulations (Hou et al., 2011; Hernandez Gonzalez et al., 2021). The four top-ranked D-peptides were purchased and tested in vitro to evaluate their inhibitory activity against $3 \mathrm{CL}^{\text {pro }}$. Remarkably, all the tested D-peptides caused $3 \mathrm{CL}^{\text {pro }}$ inhibition at $20 \mu \mathrm{M}$ during primary assays, resulting in up to $85 \%$ loss of proteolytic activity in certain cases. Therefore, the devised workflow led to promising results potentially extensible to broader applications related to protein inhibition.

\section{MATERIALS AND METHODS}

\section{Preparation of the Protein Structure for Virtual Screening and MD Simulations}

The crystal structure of free $3 \mathrm{CL}^{\text {pro }}$ (PDB: 6Y2E, resolution $1.75 \AA$ ) (Zhang et al., 2020b) was chosen to conduct SBVSs and $\mathrm{MD}$ simulations with identified D-peptides. Protonation at $\mathrm{pH} 7.2$ was performed using the PDB2PQR Web Server (https://server.poissonboltzmann.org/pdb2pqr) (Dolinsky et al., 2007). The protonated structure was then converted into the pdbqt file required for SBVS with the program prepare_receptor4. py of AutoDockTools 4 (Morris et al., 2009). MD simulations of

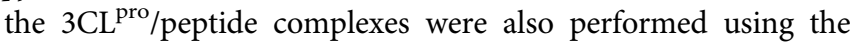
predicted protonation states of the ionizable protein residues.

\section{Building an In-House Library of D-Tripeptides and D-Tetrapeptides}

An in-house library of capped D-tripeptides and D-tetrapeptides (for brevity's sake the term "capped" will be omitted hereinafter when referring to the D-peptides) was built using tleap of 
Amber20 (Case et al., 2020). Briefly, tleap was called inside three or four nested loops, depending on the peptide length, each iterating over all the different amino acids. For HIS, its two different neutral tautomers were considered, thus totalizing 21 residue types. The sequence command of tleap was employed to create each peptide, with acetyl (ACE) and N-methyl amide (NME) capping groups being added at the $\mathrm{N}$ - and C-termini, respectively. The default $\mathrm{L}$ configuration of the $\mathrm{Ca}$ atoms was then inverted to D configuration using the flip command of tleap (Case et al., 2020). Of note, the chiral centres of ILE and THR side-chains were not inverted, thus being modeled as D-alloisoleucine and D-allo-threonine diastereomers, which will be referred to as ILE and THR hereinafter. The D-peptides were embedded into TIP3P octahedral solvation boxes, with edges spanning at least $10 \AA$ from the solute surface, and counter-ions $\left(\mathrm{Na}^{+}\right)$were added to neutralize the system net charge. Topology and coordinate files for every solvated D-peptide were finally generated and saved for subsequent steps.

The systems were subjected to two rounds of energy minimization (EM) using pmemd. MPI of Amber20 in order to obtain a suitable conformation of each D-peptide in solution (Case et al., 2020). The first EM step consisted of 500 cycles of steepest descents (SD) followed by 500 cycles of conjugate gradient (CG) minimization, and both were carried out in the presence of harmonic restraints applied to the D-peptide heavy atoms $(k=$ $10 \mathrm{kcal} \cdot \mathrm{mol}^{-1} \cdot \AA^{2}$ ). The second EM was performed with no harmonic restraints and, as before, involved 500 cycles of SD followed by 500 cycles of CG minimization. The energy-minimized D-peptides were then stripped off the solvent and counter-ions, saved as $p d b$ files with cpptraj of Amber20 (Case et al., 2020), and converted into pdbqt files with prepare_ligand4. py of AutoDock Tools 4 (Morris et al., 2009). This step completed the preparation of the peptide library containing 9,261 D-tripeptides and 194,481 D-tetrapeptides. Libraries of larger peptides were not prepared, as docking algorithms tend to produce less accurate results for molecules bearing many freely-rotatable bonds (Rentzsch and Renard, 2015; Ciemny et al., 2018; Hashemi et al., 2021).

\section{Structure-based Virtual Screening}

Despite $3 \mathrm{CL}^{\text {pro }}$ being a homodimer in solution, a monomer was chosen for SBVS and subsequent post-docking rescoring steps, as each active site in the functional homodimer is formed by residues belonging to an individual chain. A $19.5 \AA \times 18.0 \AA \mathrm{x}$ $22.0 \AA$ box spanning the whole active site of $3 \mathrm{CL}^{\text {pro }}$ (PDB: 6Y2E) was then built using the Autodock/Vina plugin of Pymol (Supplementary Figure S1), and docking of D-peptides was performed with AutoDock Vina v1.12 (DeLano, 2002; Seeliger and de Groot, 2010; Trott and Olson, 2010). Default parameters, i.e., 9 poses per ligand, the exhaustiveness of the search equal to 8 , and an energy difference of $3 \mathrm{kcal} / \mathrm{mol}$ between the best and worst poses were set for the docking simulations during SBVSs. D-tripeptide and D-tetrapeptide libraries were screened and ranked separately to reduce the impact of ligand-size bias (Chang et al., 2010). Based on the obtained AutoDock Vina scores $\left(S_{\text {vina }}\right)$, the 82 and 179 top-ranked D-tripeptides and D-tetrapeptides, respectively, were selected for subsequent rescoring steps (Figure 1).

\section{Post-docking Rescoring Steps}

The selected D-tripeptides and D-tetrapeptides underwent in parallel a series of rescoring steps involving MM-GBSA free energy calculations (Gohlke et al., 2003; Gohlke and Case, 2004; Miller et al., 2012; Case et al., 2020), docking with increased exhaustiveness of the search and MD simulations (Figure 1). First, each of the nine docking poses per selected D-peptide was rescored based on their MM-GBSA effective free energies $\left(\Delta G_{e f f}\right)$, calculated for the energy-minimized solvated complexes. The D-peptides were then re-ranked according to the $\Delta G_{\text {eff }}$ values of their respective lowest-energy poses. The best D-peptides were then docked again into the $3 \mathrm{CL}^{\text {pro }}$ active site with AutoDock Vina. For this step, the exhaustiveness of the search was increased to 80 , and four independent and randomly-initialized docking simulations, each generating 20 different poses (80 poses in total), were run for every D-peptide. As before, lowest-energy poses were identified by rescoring the 80 docking poses per ligand using their corresponding MM-GBSA $\Delta G_{\text {eff }}$ values after EM of the $3 \mathrm{CL}^{\mathrm{pro}} /$ peptide complexes. Finally, 10 ns of MD simulations, subsequently extended to $110 \mathrm{~ns}$ for the best candidates, were conducted, and average MM-GBSA $\Delta G_{\text {eff }}$ values were calculated for the generated trajectories $\left(\Delta G_{e f f, 10 n s}\right.$ and $\Delta G_{\text {eff,110ns, }}$, respectively). The D-peptides with average $\Delta G_{e f f, 110 n s}$ and Root Mean Squared Deviation (RMSD) values (see Trajectory Analyses section) lower than the indicated cutoffs were proposed as potential $3 \mathrm{CL}^{\text {pro }}$ inhibitors (Figure 1).

\section{Simulations Setup}

The $3 \mathrm{CL}^{\mathrm{pro}} /$ peptide complexes were parametrized with Amber ff14SB force-field (Maier et al., 2015) and embedded in octahedral simulation boxes with edges spanning, at least, $10 \AA$ away from the solute surface, filled with TIP3P waters (Price and Brooks, 2004) and sufficient $\mathrm{Na}^{+}$counter-ions to neutralize the systems (Supplementary Table S1) (Li et al., 2015). All previous steps were carried out with tleap of Amber20 (Case et al., 2020). Again, as individual chains form the two active sites of the $3 \mathrm{CL}^{\text {pro }}$ homodimer, $\mathrm{MD}$ simulations were carried out with monomeric $3 \mathrm{CL}^{\text {pro }}$ in complex with the selected D-peptides. This approach was deemed sufficiently accurate to assess the complex stability and significantly reduced the computational demand by decreasing the size of the simulated systems. The solvated complexes underwent two consecutive EMs identical to those conducted during the preparation of the D-peptide libraries. Each energy-minimized system was equilibrated prior to the productive run through a $1 \mathrm{~ns}$ NVT heating using a linear temperature gradient from 10 to $298.15 \mathrm{~K}$, followed by a $1 \mathrm{~ns}$ NPT equilibration at $p=1$ bar and $T=298.15 \mathrm{~K}$. Both equilibration steps were carried out in the presence of harmonic restraints $\left(k=10 \mathrm{kcal} \cdot \mathrm{mol}^{-1} \cdot \AA^{-2}\right)$ applied to the complex heavy atoms. Then, four consecutive $1 \mathrm{~ns} \mathrm{MD}$ simulations in the NPT ensemble, in which the harmonic constant was lowered from 8 to $2 \mathrm{kcal} \mathrm{mol}^{-1} \cdot \AA^{-2}$ in $2 \mathrm{kcal} \mathrm{mol}^{-1} \cdot \AA^{-2}$ strides, were carried out. Subsequently, $10 \mathrm{~ns}$ NVT MD simulations, extended to $110 \mathrm{~ns}$ for several systems showing favorable $\Delta G_{\text {eff,10ns }}$ values, were conducted. Finally, the $\mathrm{MD}$ simulations corresponding to the four experimentally tested D-peptides in complex with $3 \mathrm{CL}^{\text {pro }}$ were extended to $1 \mu \mathrm{s}$. Replicate $1 \mu \mathrm{s}$ MD simulations for these systems were also performed by subjecting the last frame of the respective $110 \mathrm{~ns}$ 


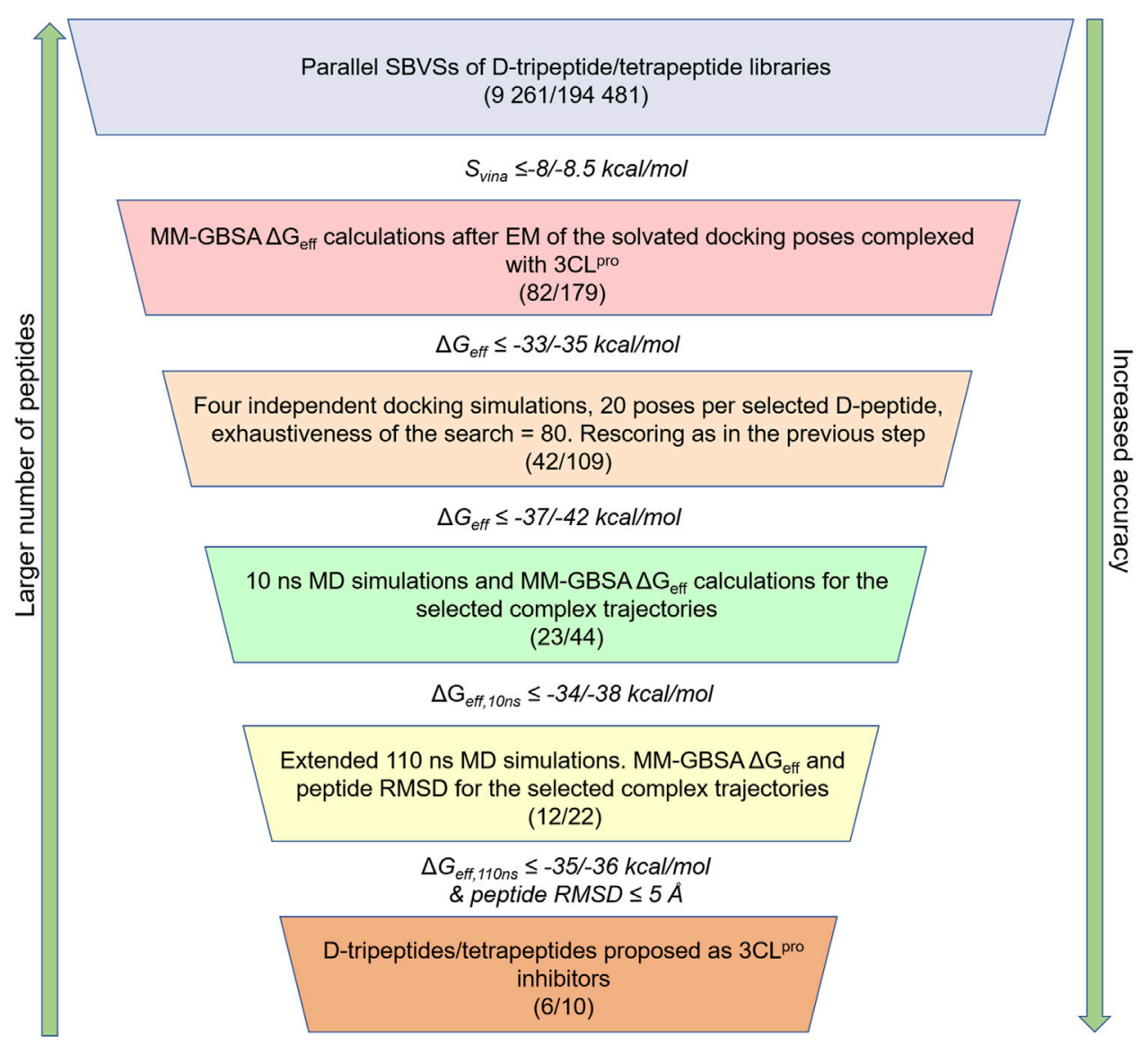

FIGURE 1 | Workflow employed for the identification of potential D-peptides targeting the $3 \mathrm{CL}^{\text {pro }}$ active site. The number of D-tripeptides and D-tetrapeptides analyzed in parallel throughout the workflow is shown in parentheses, i.e., (number of D-tripeptides/number of D-tetrapeptides). The cut-offs used to select the peptides at every step are indicated as follows: cut-off for D-tripeptides/cut-off for D-tetrapeptides. These values were set on the basis of the results, so that a manageable number of D-peptides would be selected for the next step.

trajectories to $\mathrm{EM}$, heating using randomly-initialized atomic velocities drawn from a Maxwell-Boltzmann distribution, and NPT equilibration steps, as described hereinbefore.

The program pmemd.cuda of Amber20 was used to run all MD simulations (Salomon-Ferrer et al., 2013; Case et al., 2020). Periodic boundary conditions were set during both EM and $\mathrm{MD}$ simulations, and long-range electrostatic interactions (for distances $>9 \AA$ ) were handled with the Particle Mesh Ewald (PME) algorithm (Darden et al., 1993). Temperature control was carried out using the Berendsen weak coupling algorithm (Berendsen et al., 1984) during heating and the Langevin thermostat (Schneider and Stoll, 1978), with a collision frequency of $2 \mathrm{ps}^{-1}$, during both NPT equilibration and NVT production runs. The Berendsen barostat (Berendsen et al., 1984), with a relaxation time of $2 \mathrm{ps}$, was employed to control the pressure during NPT equilibrations. Covalent bonds involving hydrogen atoms of the solute and water molecules were constrained with the SHAKE (Ryckaert et al., 1977) and SETTLE (Miyamoto and Kollman, 1992) algorithms. The equation of motion of the simulated systems was integrated using the leap-frog algorithm with a timestep of $2 \mathrm{fs}$ (Case et al., 2020).

\section{MM-GBSA Free Energy Calculations}

MM-GBSA free energy calculations were conducted with the MMPBSA.py program of Amber20 (Gohlke et al., 2003; Gohlke and Case, 2004; Miller et al., 2012; Case et al., 2020). The single trajectory approach, in which the free ligand and the free receptor trajectories are extracted from that of the complex, was adopted in all cases after stripping off the solvent and ions (Miller et al., 2012). The GB-neck2 implicit solvation model was employed to determine the polar solvation free energy component (Nguyen et al., 2013; Case et al., 2020), as it yielded a good correlation with experimental results in a set of protein-peptide complexes (Hernandez Gonzalez et al., 2017). Moreover, the calculations were performed using the mbondi3 set of atomic radii, a salt concentration of $0.1 \mathrm{M}$ and external and internal dielectric constants of 80 and 1 , respectively. The surface tension and the offset values were set to $0.0072 \mathrm{kcal} \cdot \mathrm{mol}^{-1} \cdot \AA^{-2}$ and zero, respectively, in order to estimate the nonpolar free-energy 
component from the variation of the solvent accessible surface areas (SASAs) of the interacting molecules, i.e., 3CL ${ }^{\text {pro }}$ and D-peptides (Case et al., 2020). In turn, SASA values were obtained with the Linear Combination of Pair-wise Overlaps (LCPO) algorithm included in Amber20 suite (Weiser et al., 1999; Case et al., 2020) using a probe radius of $1.4 \AA$ (Connolly, 1983). As mentioned before, the $\Delta G_{e f f, 10 n s}$ and $\Delta G_{e f f, 110 n s}$ values obtained from the 10 and $110 \mathrm{~ns}$ MD simulations after discarding the first 2 and $40 \mathrm{~ns}$, respectively, allowed us to select the most promising D-peptides at the two last steps of the workflow shown in Figure 1.

\section{Principal Component Analysis}

Principal component analysis (PCA) (Amadei et al., 1993) was carried out for the $1 \mu$ s trajectories of $3 \mathrm{CL}^{\text {pro }}$ in complex with the experimentally-tested D-peptides. Using this technique, we sought to reduce the phase space dimensionality by projecting the system's motion along the two eigenvectors, known as principal components (PCs), of the highest variance (=largest eigenvalues), as our main interest here was to identify the different conformations of the $\mathrm{D}$-peptides in the $3 \mathrm{CL}^{\text {pro }}$ active site during the long MD simulations. PCA was performed only for the $\mathrm{C} a$ atoms of the former molecules. The replicate $1 \mu \mathrm{MD}$ simulations of each system were concatenated and fitted using cpptraj of Amber 20 (Case et al., 2020). Trajectory fitting was carried out concerning the $3 \mathrm{CL}^{\text {pro }}$ backbone atoms belonging to the chymotrypsin-like (ChT-like) domains, i.e., domains I and II, residues 8 to 183 (Tahir Ul Qamar et al., 2020), which contain the active site. This step ensured that the displacements of the D-peptide $\mathrm{Ca}$ atoms during the long $\mathrm{MD}$ simulations were measured relative to the enzyme's active site and eliminated the influence of domain III motions during fitting. The program gmx covar of Gromacs v.5.1.4 (Abraham et al., 2015) was employed to calculate the covariance matrices of the D-peptide Ca positions along the fitted trajectories and their corresponding sets of eigenvalues and eigenvectors. Twodimensional (2D) projections of the trajectories onto the first two eigenvectors (PC1 and PC2) were obtained with gmx anaeig of Gromacs v5.1.4.

PCA was combined with free energy landscape (FEL) visualization and clustering to determine central structures for the different conformations sampled during the MD simulations (Papaleo et al., 2009). FELs were obtained from the 2D projections and depicted as heatmaps by discretizing the $2 \mathrm{D}$ phase space into 700 square bins of equal size and counting the number of points within each. The free energy value corresponding to bin $i\left(\Delta G_{i}\right)$ was then calculated from the probability of finding the system into that bin $\left(p_{i}\right)$ using the equation:

$$
\Delta G_{i}=-R T \ln \left(p_{i}\right)
$$

where $R$ is the gas constant and $T$, the temperature $(298.15 \mathrm{~K})$.

Finally, the trajectories were split using the K-means algorithm with random initial seeds, implemented as an option of the cluster command of cpptraj (Case et al., 2020), by using the PC1 and PC2 values as a metric. The number of clusters in each case was set to the main FEL basins observed in the corresponding heatmap.
Each newly generated trajectory was subsequently clustered to determine its central structure through RMSD clustering (see next section for details).

\section{Trajectory Analyses}

The 110 ns MD simulations were clustered in order to select the central structure of each analyzed $3 \mathrm{CL}^{\mathrm{pro}} /$ peptide complex. This step was performed with the cluster command of cpptraj using the average linkage algorithm (Shao et al., 2007; Case et al., 2020). The RMSD for the heavy atoms of the peptide and $3 \mathrm{CL}^{\text {pro }}$ residues lying within a $4 \AA$ cut-off was chosen as a metric for clustering the trajectories. This procedure was also applied to determine the central structures corresponding to the main energy minima observed in the $\mathrm{PC} 1$ vs $\mathrm{PC} 2$ projections of the complexes subjected to PCA. The main central structures were selected for structural representation using Pymol 2.1.0 (DeLano, 2002). RMSD values for the D-peptide heavy atoms along the 110 ns trajectories were calculated with rms of cpptraj after fitting all frames with respect to the $3 \mathrm{CL}^{\text {pro }}$ backbone atoms belonging to the ChT-like domains in the corresponding starting structures $(t=0)$. These RMSD values were averaged during the last $20 \mathrm{~ns}$ of the trajectories to assess whether the binding modes of the D-peptides sampled at the end of the MD simulations deviated significantly from those of the starting structures. Root mean square fluctuations (RMSFs) were calculated with the rmsf command of cpptraj (Case et al., 2020). Finally, hydrogen bonds (H-bonds) formed at the complex interfaces during the MD simulations were determined with hbond command of cpptraj (Case et al., 2020), using the following geometric criteria: a donor-acceptor distance $\leq 3.5 \AA$ and a donor-Hacceptor angle $\geq 120^{\circ}$.

\section{D-Peptide Synthesis}

Synthetic D-enantiomeric peptides used in this study (4P1, 4P2, $4 \mathrm{P} 3$, and 4P4) were synthesized by Genscript (Leiden, NL), with a purity of $\geq 90 \%$. The D-peptides were acetylated at the $\mathrm{N}$-terminus and methylated at the C-terminus.

\section{Cloning, Expression, and Purification of SARS-CoV-2 $3 \mathrm{CL}^{\text {pro }}$}

The codon-optimized cDNA encoding SARS-CoV-2 $3 \mathrm{CL}^{\text {pro }}$ (Uniprot entry: P0DTD1, virus strain: hCoV-19/Wuhan/ WIV04/2019) was synthesized and implemented in the ampicillin-resistant vector pGEX-6P-3 (BioCat $\mathrm{GmbH}$, Heidelberg, Germany). The construct contains an N-terminal GST-tag and a PreScission protease cleavage site (LEFLFQGP). Expression and purification were performed as described before (Eberle et al., 2021).

\section{Primary $3 \mathrm{CL}^{\text {pro }}$ Enzymatic Inhibition Assay}

All measurements were performed in triplicate in $20 \mathrm{mM}$ Tris $\mathrm{pH}$ 7.2, $200 \mathrm{mM} \mathrm{NaCl}, 1 \mathrm{mM}$ EDTA, and $1 \mathrm{mM}$ TCEP as described previously (Zhang et al., 2020a; Zhang et al., 2020b; Ma et al., 2020; Eberle et al., 2021). $20 \mu \mathrm{M}$ of the peptides (4P1, 4P2, 4P3, and 4P4) were pipetted into a Corning 96-Well plate (Sigma Aldrich), $3 \mathrm{CL}^{\text {pro }}$ was added to a final concentration of $500 \mathrm{nM}$, 

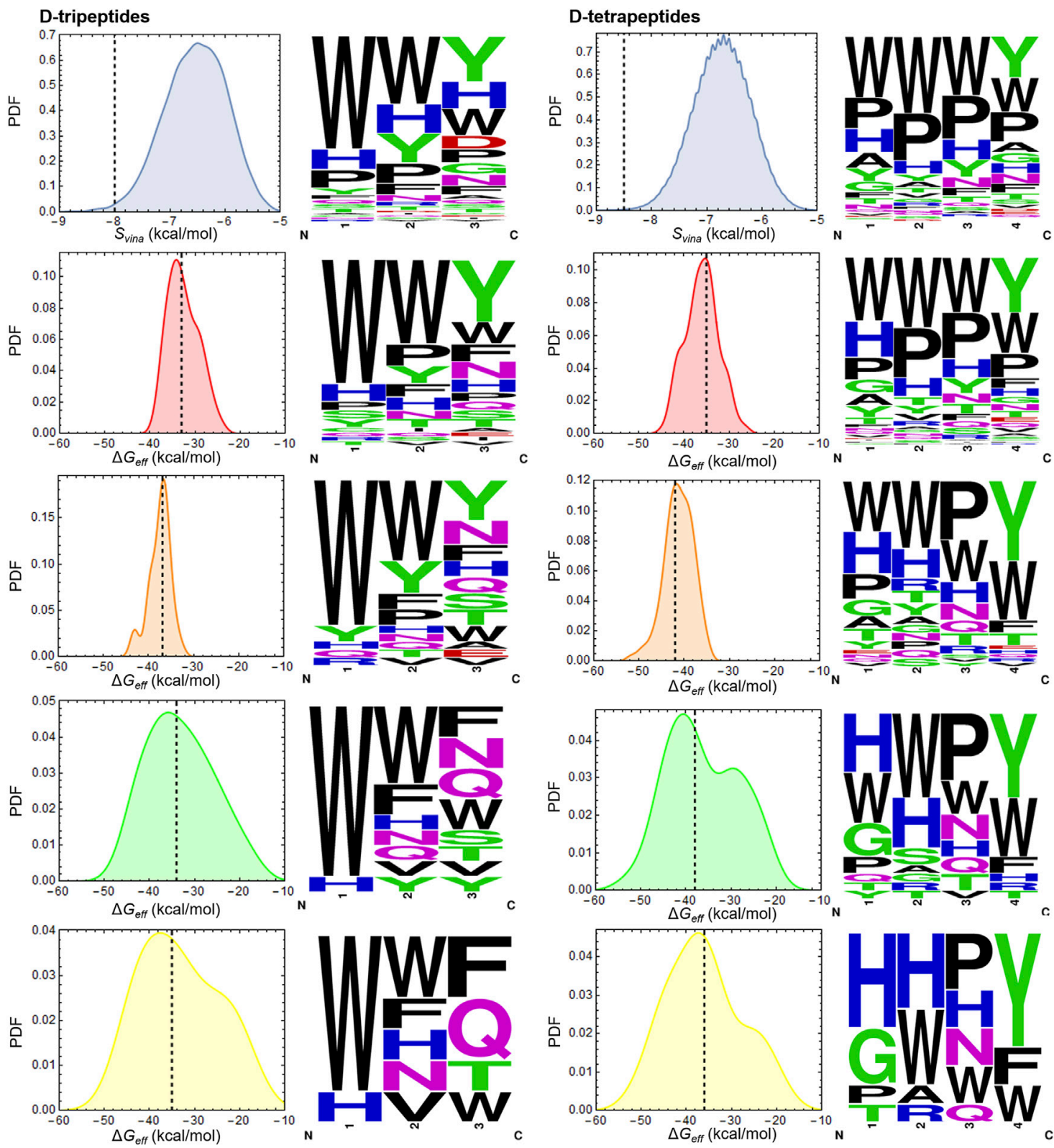

FIGURE 2 | Energy distributions and D-peptide sequences at every step of the in silico workflow. Each histogram is colored as the corresponding step of the workflow shown in Figure 1. Dashed lines indicate the cut-off values employed to select the best candidates at every step. Peptides with $S_{\text {vina }} / \Delta G_{\text {eff }}$ values to the left of the dashed lines were selected. PDF is the abbreviation for probability density function. Sequence logos of the D-peptides identified at every step are shown to the right of the corresponding histograms. Letter sizes are proportional to the frequency of occurrence of the indicated residues in each position. $\mathrm{N}$ and $\mathrm{C}$ below the graphs stand for the N- and C- termini, respectively. Sequence logos were generated at https://weblogo.berkeley.edu/logo.cgi. A D-tetrapeptide was excluded as potential $3 \mathrm{CL}^{\text {pro }}$ inhibitor after filtering according to the RMSD value in the last rescoring step (Figure 1, not shown). The last sequence logo of D-tetrapeptides does not contain the excluded peptide.

and the mixture was incubated for $30 \mathrm{~min}$. Subsequently, the enzymatic reaction was initiated by adding the fluorogenic substrate DABCYL-KTSAVLQ $\downarrow$ SGFRKME-EDANS (Bachem, Switzerland) to a final concentration of $50 \mu \mathrm{M}$. The gradual release of fluorescent 5-((2-Aminoethyl)amino)naphthalene-1-sulfonic acid (EDANS) was monitored for $30 \mathrm{~min}$ with $60 \mathrm{~s}$ intervals. The excitation and emission wavelengths were 360 and $460 \mathrm{~nm}$, respectively, using an Infinite 200 PRO plate reader (Tecan, Männedorf, Switzerland). The temperature was set to $37^{\circ} \mathrm{C}$. The results are shown as mean value \pm standard deviation (STD).

\section{Statistical Analyses}

Block averaging was conducted using gmx analyze of Gromacs v.5.1.4 (Hess, 2002; Abraham et al., 2015) to estimate standard errors of the mean (SEMs) from time-dependent values collected from MD simulations, such as the reported $\Delta G_{\text {eff,110ns }}$ and RMSD 
TABLE 1 | D-peptides identified in silico as potential $3 \mathrm{CL}^{\text {pro }}$ inhibitors.

\begin{tabular}{|c|c|c|c|}
\hline Peptide ID & Peptide sequence $^{a}$ & $\Delta G_{\text {eff, } 11 \text { ons }}(\mathrm{kcal} / \mathrm{mol})^{\mathrm{b}}$ & $\operatorname{RMSD}(\AA ̊ \cap){ }^{c}$ \\
\hline $3 \mathrm{P} 1$ & ACE-TRP-TRP-THR-NME & $-44.2 \pm 0.5$ & $2.58 \pm 0.03$ \\
\hline 3Р3 & ACE-TRP-PHE-GLN-NME & $-40.4 \pm 2.0$ & $4.22 \pm 0.05$ \\
\hline $3 \mathrm{P} 4$ & ACE-TRP-VAL-PHE-NME & $-40.3 \pm 1.3$ & $2.3 \pm 0.3$ \\
\hline 3P5 & ACE-TRP-TRP-GLN-NME & $-36.5 \pm 0.6$ & $4.43 \pm 0.04$ \\
\hline $4 \mathrm{P} 1$ & ACE-GLY-TRP-ASN-TYR-NME & $-50.2 \pm 0.9$ & $3.60 \pm 0.04$ \\
\hline $4 \mathrm{P} 2$ & ACE-GLY-TRP-HIE-TRP-NME & $-45.6 \pm 0.7$ & $3.0 \pm 0.9$ \\
\hline $4 \mathrm{P} 3$ & ACE-HIE-ALA-PRO-TRP-NME & $-44.9 \pm 0.9$ & $1.17 \pm 0.05$ \\
\hline $4 \mathrm{P} 4$ & ACE-HIE-HIE-PRO-TYR-NME & $-44.9 \pm 1.5$ & $2.70 \pm 0.09$ \\
\hline 4P5 & ACE-THR-HIE-TRP-TYR-NME & $-44.5 \pm 2.0$ & $3.574 \pm 0.08$ \\
\hline $4 \mathrm{P} 6$ & ACE-HIE-HIE-ASN-TYR-NME & $-42.8 \pm 0.9$ & $3.20 \pm 0.04$ \\
\hline
\end{tabular}

${ }^{a}$ Residues are shown in three-letter code and separated by hyphens. ACE and NME are the N-and C-terminal caps added to the D-peptides. HIE and HID are HIS tautomers. ${ }^{b} M M$-GBSA average effective free energies calculated over the last $70 \mathrm{~ns}$ of each $110 \mathrm{~ns}$ MD trajectory $\pm S E M s$ estimated through block averaging. See instantaneous $\triangle G_{\text {eff }} v a l u e s ~ v s$ time plots for every system along their respective 110 ns MD simulations in Supplementary Figure S2.

${ }^{\circ}$ Mean RMSD values for the peptide heavy atoms with respect to the starting structure $(\mathrm{t}=0)$ calculated over the last $20 \mathrm{~ns}$ of each $110 \mathrm{~ns} \mathrm{MD}$ trajectory \pm standard errors of the mean estimated through block averaging. See RMSD values vs time plots for all systems along their respective 110 ns MD simulation in Supplementary Figure S3.

mean values. On the other hand, the statistical significance of the residual activity mean values' differences was performed with GraphPad Prism software version 8 (GraphPad, 2018) and was assessed with one-way analyses of variance (ANOVA), followed by Tukeys' multiple comparison test. Significant differences were considered at $p<0.01\left(^{* *}\right)$ and $p<0.001\left(^{* * *}\right)$.

\section{RESULTS}

\section{D-Peptides Predicted as $3 \mathrm{CL}^{\text {pro }}$ Inhibitors}

The D-peptides proposed as potential $3 \mathrm{CL}^{\text {pro }}$ inhibitors were selected through a workflow involving SBVS and several rescoring steps (Figures 1, 2). The top-ranked D-peptides, according to the $S_{\text {vina }}$ values, were made up mainly of aromatic and hydrophobic residues, with TRP being the residue most frequently found in all positions except the C-terminus, in which TYR was the most abundant (Figure 2). Certain regions of $3 \mathrm{CL}^{\text {pro }}$ active site, such as the $\mathrm{S} 2$ pocket, are hydrophobic (Jin et al., 2020), which can favor the binding of peptides containing the aforementioned residues. However, the already known bias of AutoDock Vina and other docking algorithms toward larger compounds can also be at play here (Pan et al., 2003; Chang et al., 2010). The subsequent rescoring steps aimed to correct this bias and enrich selected D-peptides' lists with accurate hits. In fact, it can be observed from the sequence logos, shown in Figure 2, that even though TRP was still prevalent at different positions of the $\mathrm{D}$-peptides, other residues became progressively more abundant throughout the workflow steps, which was particularly apparent for D-tetrapeptides, in which HIS and PRO were found to be predominant in positions 1 to 3 after completing the workflow (Figure 2).
Apart from HIS, other residues with polar side-chains, such as ASN, GLN, THR, and ARG, occurred more frequently among the $D$-peptides selected after the last rescoring step than among those selected from the SBVS. Consequently, the D-peptides prioritized after the workflow completion were, on average, smaller in size than those ranked in the top positions by the docking algorithm. Overall, the rescoring steps tended to upweight the occurrence of intermolecular $\mathrm{H}$-bonds mediated by the side-chains of $\mathrm{D}$-peptide residues at the expense of ligand size. These results, in turn, suggest a reduced impact of ligand-size bias on the final set of chosen D-peptides. The D-tripeptides and D-tetrapeptides identified as potential $3 \mathrm{CL}^{\text {pro }}$ inhibitors are shown in Table 1. All these D-peptides fulfilled the conditions set throughout the workflow steps. They showed a good affinity for the enzyme and stability of the initial docking pose during the MD simulations, measured in terms of $\Delta G_{\text {eff,110ns }}$ and peptide RMSD mean values, respectively (Table 1 and Supplementary Figures S2, S3).

\section{Structural Features of the Predicted $3 \mathrm{CL}^{\text {pro/ }}$ D-peptide Interfaces}

The central structures of the selected D-peptides (Table 1) in complex with $3 \mathrm{CL}^{\text {pro }}$ obtained after clustering the respective 110 ns $\mathrm{MD}$ simulations are shown in cartoon representation in Figure 3. Moreover, for comparison purposes, the L-peptide VTLQSK (L-Pep) is depicted at the $3 \mathrm{CL}^{\text {pro }}$ active site (Figure 3). L-Pep corresponds to the C-terminus of the homologue SARS-CoV $3 \mathrm{CL}^{\text {pro }}$ and inserts into the active site of a neighboring protease chain in the $\mathrm{PDB}$ structure $5 \mathrm{~B} 6 \mathrm{O}$ (Muramatsu et al., 2016), thus allowing the template-based modeling of the SARS-CoV-2 $3 \mathrm{CL}^{\mathrm{pro}} / \mathrm{L}-\mathrm{Pep}$ complex. Of note, 

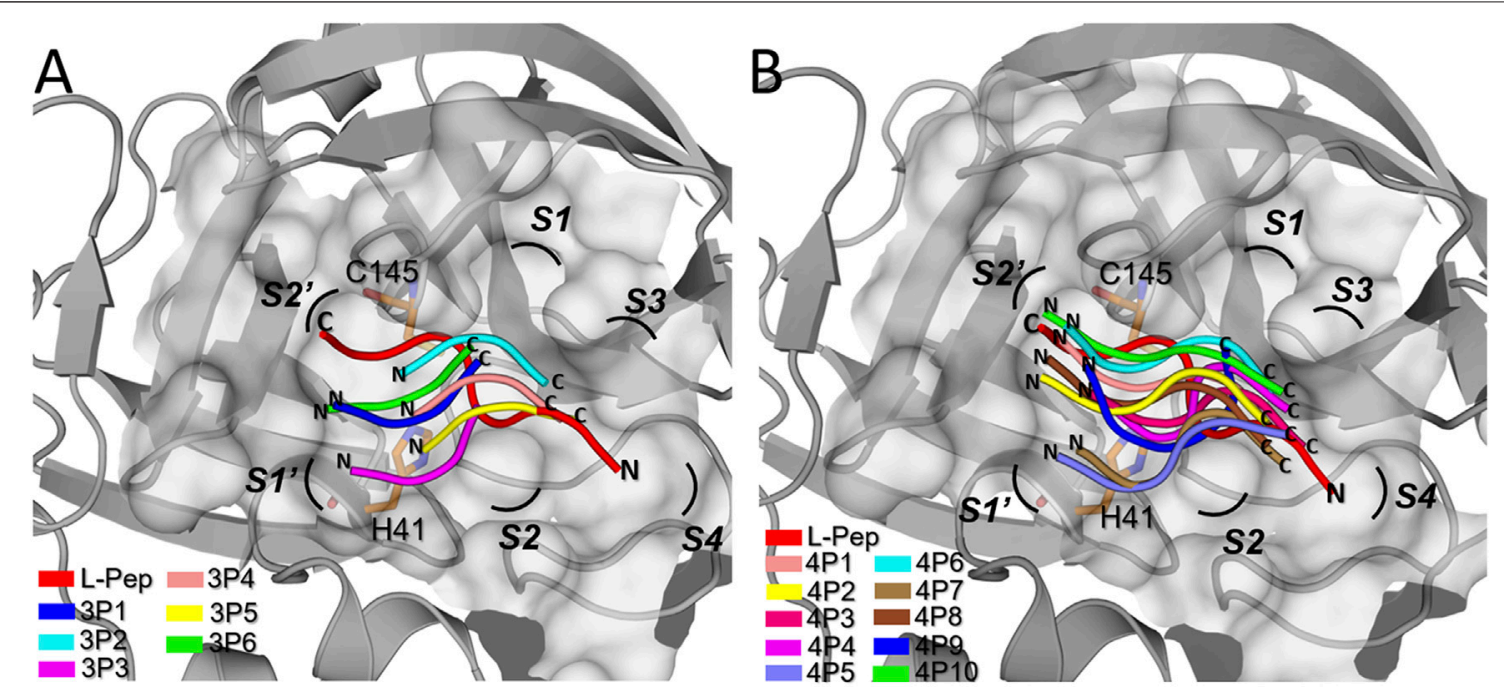

FIGURE 3 | Accommodation of the D-peptide backbones into the active site of $3 \mathrm{CL}^{\text {pro }}$. Superimposed central structures of $3 \mathrm{CL}^{\text {pro }}$ in $\mathrm{COmplex}$ with the (A) D-tripeptides and (B) D-tetrapeptides proposed as potential inhibitors (Table 1), determined from the respective 110 ns MD simulations. In both cases, the crystal structure of $3 \mathrm{CL}^{\text {pro }}$ (PDB: $6 \mathrm{Y} 2 \mathrm{E}$ ) is depicted in gray and the catalytic residues, $\mathrm{H} 41$ and $\mathrm{C} 145$, are shown as orange sticks. The protease active site is represented as a gray surface. The D-peptides of the structurally-aligned complexes are colored differently (see legends). The $3 \mathrm{CL}^{\text {pro }} \mathrm{Conformation}$ of each central structure was omitted for clarity's sake. For comparison purposes, each panel shows the conformation of the L-peptide VTLQSK (L-Pep) into SARS-CoV-2 3CL ${ }^{\text {pro }}$, obtained through structural alignment between the former protease and the crystal structure of SARS-CoV 3CL ${ }^{\text {pro }}$ (PDB: 5B6O) (Muramatsu, et al., 2016). L-Pep corresponds to the $\mathrm{C}$-terminal portion of a neighboring SARS-CoV $3 \mathrm{CL}^{\text {pro }}$ chain that inserts in a substrate-like conformation into the protease active site. Letters $\mathrm{N}$ and $\mathrm{C}$ indicate the $\mathrm{N}$ - and C-termini of the peptides, respectively. The $3 \mathrm{CL}^{\text {pro }}$ subsites $\mathrm{S} 2$ ' to $\mathrm{S} 4$ are labeled accordingly.

the backbones of all the analyzed D-peptides adopt an orientation opposite (=retro-binding) to that of L-Pep (Figures 3A,B). As we will show below in more detail, the retro-binding enables the formation of key interactions between the active site residues and the backbone atoms of the D-peptide present at the $3 \mathrm{CL}^{\mathrm{pro}} / \mathrm{L}-\mathrm{Pep}$ interface.

It can be seen that there is a partial overlap between the L-Pep and D-peptide backbones, especially involving the $\mathrm{N}$ - and C-terminal portions of L-pep and D-tetrapeptides, respectively (Figure 3). Therefore, several key interactions mediated by the peptide backbones and neighboring residues on the $S$ side of the $3 \mathrm{CL}^{\text {pro }}$ active site are expected to be preserved in the predicted complexes. The D-tetrapeptide backbones span a larger stretch of the $3 \mathrm{CL}^{\text {pro }}$ active site when compared to the D-tripeptides and resemble, to a greater extent, the overall accommodation of L-Pep, which could explain, in turn, the generally more favorable free energy values obtained for the D-tetrapeptides in complex with $3 \mathrm{CL}^{\text {pro }}$ (Table 1). On the other hand, the backbone accommodation was more divergent in the $\mathrm{N}$ terminal regions of the $\mathrm{D}$-peptides, not only relative to L-pep but among themselves (Figure 3). This might arise from the plasticity of the $3 \mathrm{CL}^{\text {pro }}$ active site (Kneller et al., 2020) and the inability of the small D-peptides to satisfy interactions equivalent to those observed for L-peptides.

The number of intermolecular H-bonds formed along the MD simulations can be used to indicate complex stability (Lokhande et al., 2021). Therefore, we decided to calculate the time profiles of such interactions in the predicted $3 \mathrm{CL}^{\mathrm{pro}} / \mathrm{D}$-peptide complexes (Supplementary Figure S4). The graphs show that all the D-peptides in Table 1 formed several $\mathrm{H}$-bonds with $3 \mathrm{CL}^{\text {pro }}$ active site residues during the $110 \mathrm{~ns} \mathrm{MD}$ simulations of the complexes (Supplementary Figure S4). The average number of intermolecular $\mathrm{H}$-bonds ranged from 3 to 8 , depending on the complex. This result underscores the good complementarity of the identified D-peptides to the $3 \mathrm{CL}^{\text {pro }}$ active site.

The analysis of the interactions occurring at the $3 \mathrm{CL}^{\mathrm{pro}} / \mathrm{L}-\mathrm{Pep}$ complex provides valuable information to study the $3 \mathrm{CL}^{\text {pro }}$ D-peptide interfaces. Therefore, a detailed structural representation of this complex was included in Figure 4. L-Pep extends along with the S4 to S2 ${ }^{\prime}$ subsites of the enzyme, displaying the $\mathrm{N}$-terminal VAL residue at position $\mathrm{P} 4$ and the C-terminal LYS at P2'. THR at P3 is exposed mainly to the solvent, whereas LEU and GLN at P2 and P1, respectively, insert into well-defined pockets. As can be observed, several amide nitrogen $(\mathrm{N})$ and carbonyl oxygen $(\mathrm{O})$ atoms of L-Pep backbone engage in $\mathrm{H}$-bond formation with $3 \mathrm{CL}^{\text {pro }}$ residues, e.g., $\mathrm{VAL}(\mathrm{N})$ T190(O), THR(N)-E166(O), THR(O)-E166(N), GLN(N)H164(O), GLN(O)-C145(N), GLN(O)-G143(N), and LYS(N)T26(O) (Figure 4). Of note, the amide oxygen (OE1) of L-Pep GLN side-chain forms a key $\mathrm{H}$-bond with the protonated $\mathrm{N}$ atom of H163 imidazole ring H163(NE2), which helps explain the preference of $3 \mathrm{CL}^{\text {pro }}$ for the former residue at P1 (Singh et al., 2020). Other H-bonds mediated by the side-chains of L-Pep residues are GLN(NE2)-F140(O) and LYS(NZ)-G143(O) at the $\mathrm{S} 1$ and $\mathrm{S} 2{ }^{\prime}$ subsites, respectively (Figure 4).

Structural representations of the interfaces of $3 \mathrm{CL}^{\text {pro }}$ in complex with the top-ranked D-tripeptides (Table 1) are shown in Figure 4. For brevity's sake, only the complexes having $\Delta G_{\text {eff } 110 n s}$ values $<-40 \mathrm{kcal} / \mathrm{mol}$ were included in the figure (Table 1). The backbone polar atoms of the D-tripeptides 


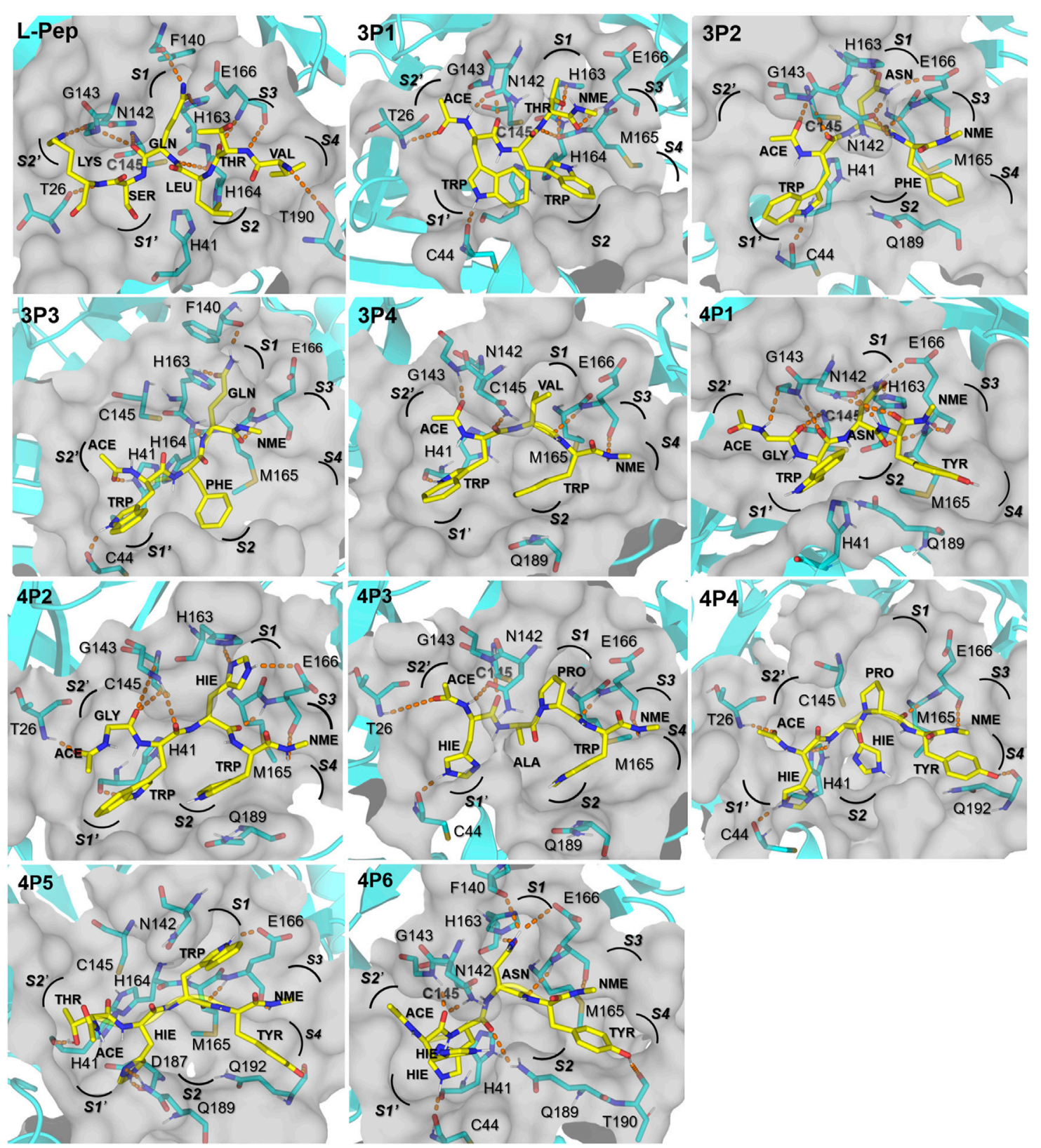

FIGURE 4 | Structural representation of top-ranked D-tripeptides and D-tetrapeptides in complex with $3 \mathrm{CL}^{\text {pro }}$. All D-peptides are shown as yellow sticks and their residues are labeled in bold and in the three-letter code. $3 \mathrm{CL}^{\text {pro }}$ residues forming $\mathrm{H}$-bonds with the peptides plus the catalytic residues $\mathrm{H} 41$ and $\mathrm{C} 145$ are labeled and represented as cyan sticks. The $3 \mathrm{CL}^{\text {pro }}$ active site cavity is depicted as a transparent gray surface. $\mathrm{H}$-bonds between the $\mathrm{D}$-peptides and the $3 \mathrm{CL}$ pro residues with occupancies $>25 \%$ during the respective 110 ns MD simulations are displayed as orange dashed lines. Subsites S4 to S2' are labeled in bold and italic. Structural representations of the remaining D-peptides included in Table $\mathbf{1}$ can be found in Supplementary Figure S5.

form a network of $\mathrm{H}$-bonds with $3 \mathrm{CL}^{\text {pro }}$ residues, some of them equivalent to those observed at the interface of the L-Pep complex. For example, the H-bond involving an $\mathrm{O}$ atom of the D-tripeptides and E166 $(\mathrm{N})$ occurs in all the analyzed interfaces. An additional H-bond between a D-tripeptide $\mathrm{N}$ atom and $\mathrm{E} 166(\mathrm{O})$ was found in the $3 \mathrm{P} 2$ and $3 \mathrm{P} 4$ complexes (Figure 4). H-bonds mediated by G143(N) and $\mathrm{C} 145(\mathrm{~N})$ and a $\mathrm{D}$-tripeptide backbone $\mathrm{O}$ atom also occur in most depicted complexes. Differently from the L-Pep complex, the amide group of N142 can form H-bonds with polar atoms of 3P1, $3 \mathrm{P} 2$, and $3 \mathrm{P} 4$ backbone, thus providing additional stabilizing interactions. This result underscores that the flexible side-chain of N142 can adopt conformations in solution that facilitate transient $\mathrm{H}$-bonding to the ligands.

Three top-ranked D-tripeptides (3P1, 3P3, and 3P4) possess aromatic residues TRP or PHE inserted into the S2 pocket of 
$3 \mathrm{CL}^{\text {pro }}$, which prefers hydrophobic residues (Chuck et al., 2010; Rut et al., 2021), whereas PHE of 3P2 occupies the S4 subsite (Figure 4). The $\mathrm{S} 1$ subsite accommodates the D-tripeptide residues THR, ASN, GLN, and VAL. Of note, ASN(OD1) and GLN(OE1) of 3P2 and 3P3, respectively, form the key H-bond with H163(NE2) observed for L-Pep GLN (Figure 4). The ASN side-chain can mimic the interactions established by the L-Pep GLN amide group because the backbone of 3P2 leans toward the entrance of the S1 pocket, thus shortening the distance to reach the bottom of this subsite (Figure 4). Like L-Pep GLN, the amide group of 3P2 ASN and 3P3 GLN can form additional H-bonds at $\mathrm{S} 1$, such as $\mathrm{ASN}(\mathrm{ND} 2) / \mathrm{GLN}(\mathrm{NE} 2)-\mathrm{F} 140(\mathrm{O})$ or $\mathrm{ASN}(\mathrm{ND} 2) /$ GLN(NE2)-E166(OE1,2). Interestingly, the hydroxyl oxygen of 3P1 THR, THR (OG1), is also able to interact with H163(NE2), which could explain the favorable $\Delta G_{\text {eff, } 110 n s}$ value obtained for this D-peptide (Table 1) despite not bearing GLN or ASN at P1. Finally, it is worth noting that all the D-tripeptides proposed as $3 \mathrm{CL}^{\text {pro }}$ inhibitors possess TRP at the N-terminus, except for 3P6 that contains HIE (Table 1). Our results indicate that TRP accommodates favorably at the $\mathrm{S}^{\prime}$ pocket, and the nitrogen of the indole group atom (NE1) can form H-bonds with C44(O)/ $\mathrm{H} 41(\mathrm{O})$ (Figure 4).

As done for the D-tripeptides, the interfaces of the top-ranked D-tetrapeptides $\left(\Delta G_{\text {eff,110ns }}<-40 \mathrm{kcal} / \mathrm{mol}\right.$, Table 1) in complex with $3 \mathrm{CL}^{\text {pro }}$ are depicted in Figure 4. The backbone of the selected D-tetrapeptides span the $3 \mathrm{CL}^{\text {pro }}$ active site from the S4 to the S2' subsites and establish several polar interactions with the neighboring residues. Key H-bonds with E166(N) and E166(O), observed in the $3 \mathrm{CL}^{\mathrm{pro}} / \mathrm{L}-\mathrm{Pep}$ complex, occur in all the analyzed D-tetrapeptide complexes. Other H-bonds, such as those involving C145(N), G143(N), the N142 side-chain amide group, T26(N) and Q192(NE2), can be found at various interfaces (Figure 4).

Four out of the six top-ranked D-tetrapeptides, i.e., 4P1, 4P4, 4P5, and 4P6, contain TYR at the C-terminus, the most abundant residue occurring at this position in the whole set of identified D-tetrapeptides (Figure 2 and Table 1). The predicted structures suggest that TYR accommodates at the S4 subsite, where the aromatic ring sits on the pocket base, mainly formed by M165 and Q192, and the side-chain hydroxyl oxygen, TYR $(\mathrm{OH})$, can form H-bonds with T190(O)/Q192(O) (Figure 4). The other two D-tetrapeptides, $4 \mathrm{P} 2$ and 4P3, insert their C-terminal TRP residues into the $\mathrm{S} 2$ pocket of $3 \mathrm{CL}^{\text {pro }}$. The latter $\mathrm{D}$-peptide also accommodates its second residue, ALA, on the opposite side of the same pocket. Likewise, $4 \mathrm{P} 4$ can accommodate its second residue, HIE2, at the S2 pocket (Figure 4). A closer look at their sequences and the predicted structures of their complexes with $3 \mathrm{CL}^{\text {pro }}$ reveals that $\mathrm{PRO}$ at $\mathrm{P} 1$ of $4 \mathrm{P} 3$ and $4 \mathrm{P} 4$ bends the $\mathrm{D}$-peptide backbones in a way that makes it feasible for upstream residues to interact with $\mathrm{S} 2$. Like PRO, ASN was found at the S1 pocket of $3 \mathrm{CL}^{\text {pro }}$ in complex with two other D-tetrapeptides, i.e., 4P1 and 4P6 (Figure 4). However, unlike the former residue, which leaves the S1 pocket largely unoccupied, ASN can form $\mathrm{H}$-bonds equivalent to L-Pep GLN. At the same position, 4P2 HIE interacts with H163(NE2) and E166(OE1,2). Of note, HIS is the second most favorable residue at $\mathrm{P} 1$ according to substrate specificity profiling conducted for SARS-CoV $3 \mathrm{CL}^{\text {pro }}$ (Chuck et al., 2010). However, to reach the bottom of the S1 pocket,

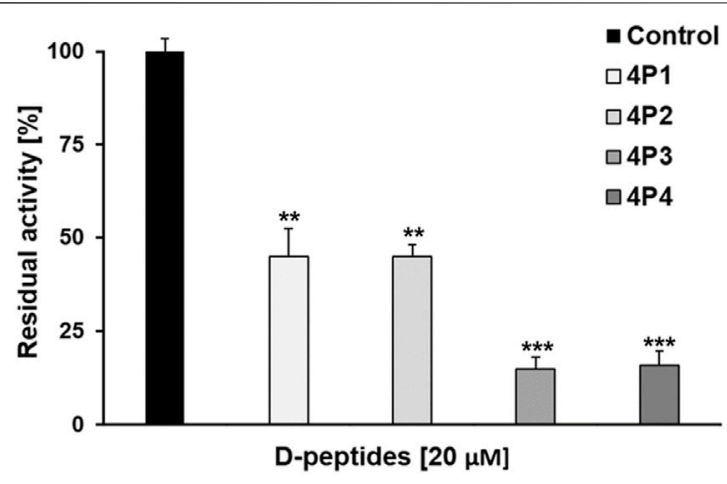

FIGURE 5 | Preliminary inhibition tests of $4 \mathrm{P} 1-4$ against $3 \mathrm{CL}^{\text {pro }} .4 \mathrm{P} 1$ and 4P2 inhibit the virus protease activity by more than 60\%. 4P3 and 4P4 inhibit the virus protease activity more than $80 \%$. Data shown are the mean \pm STD from three independent measurements $(n=3)$. Asterisks mean that the data differs from the control $\left(0 \mu \mathrm{M}\right.$ inhibitor) significantly at $\left.p<0.01{ }^{(*}\right)$ and $p<$ $\left.0.001{ }^{\star \star \star}\right)$, level according to ANOVA and Tukey's test.

HIS, like ASN, requires a backbone accommodation closer to the pocket entrance, which is accessible to the $\mathrm{D}$-peptides according to our predictions. The specificity for HIS at P1 in L-peptides has been explained by proposing that this residue interacts with the N142 side-chain (Chuck et al., 2010). TRP was also found at the $\mathrm{S} 1$ subsite of the $3 \mathrm{CL}^{\mathrm{pro}} / 4 \mathrm{P} 5$ complex (Figure 4). In this case, the residue does not penetrate deeply into the subsite but forms the H-bond TRP(NE1)-E166(OE1,2).

HIE and TRP, in that order, are the most abundant residues of the top-ranked D-tetrapeptides placed at the $\mathrm{S1}^{\prime}$ subsite (Figure 4). HIE (NE2) is capable of forming H-bonds with $\mathrm{C} 44(\mathrm{O})$ at the interfaces of $3 \mathrm{CL}^{\text {pro }}$ in complex with $4 \mathrm{P} 3,4 \mathrm{P} 4$, and 4 P6 or with Q189(NE2) and D187(O) at the interface of the $3 \mathrm{CL}^{\mathrm{pro}} / 4 \mathrm{P} 5$ complex. Interestingly, the latter $\mathrm{D}$-peptide is the only one bearing an N-terminal THR residue, which forms $\mathrm{H}$-bonds with $\mathrm{H} 41(\mathrm{O})$. In this case, the ACE cap is sticking out to the solvent instead of lying at the $\mathrm{S} 22^{\prime}$ subsite, as in the remaining complexes of the identified D-tetrapeptides (Figure 4).

\section{In vitro Inhibitory Activity of the Four Top-Ranked D-Tetrapeptides Against $3 C L^{\text {pro }}$}

The energetic and structural analyses presented in the previous sections demonstrated that the selected D-tetrapeptides displayed, in general, more favorable free energy values and better complementarity with the $3 \mathrm{CL}^{\text {pro }}$ active site than D-tripeptides (Table 1 and Figures 3, 4). Therefore, to assess the validity of the computational workflow for D-peptide identification, we decided to test in vitro the inhibitory activity of the four top-ranked D-tetrapeptides, $4 \mathrm{P} 1$ to $4 \mathrm{P} 4$ (Table 1).

The results of the primary screening are shown in Figure 5. All the D-tetrapeptides, tested at a final concentration of $20 \mu \mathrm{M}$, significantly reduced the activity of $3 \mathrm{CL}^{\text {pro }}$ relative to the control assay. In fact, the percentage of residual activity of the protease dropped below $20 \%$ upon incubation with $4 \mathrm{P} 3$ and $4 \mathrm{P} 4$, which are the most potent $\mathrm{D}$-peptides. On the other hand, both $4 \mathrm{P} 1$ and 
$4 \mathrm{P} 2$ inhibited roughly $55 \%$ of the enzymatic activity under such conditions. Our results indicate that the devised computational workflow successfully identified promising D-peptides displaying inhibitory potency against $3 \mathrm{CL}^{\text {pro }}$ in the micromolar concentration range.

\section{Identification of the Most Stable Binding Modes of 4P1, 4P2, 4P3, and 4P4 to $3 \mathrm{CL}^{\text {pro }}$ Combining Long MD Simulations, PCA, FEL and Clustering}

Replicate $1 \mu$ s $\mathrm{MD}$ simulations were conducted for $3 \mathrm{CL}^{\text {pro }}$ in complex with the experimentally-tested D-tetrapeptides to assess the time stability of binding modes previously proposed from shorter MD simulations (Figure 4) and the possible occurrence of alternate bound conformations. First, we noticed large deviations in the RMSD values for all $3 \mathrm{CL}^{\text {pro }}$ backbone atoms concerning the starting structures along several $1 \mu$ s trajectories (Supplementary Figure S6). However, it became apparent through visual inspection that such deviations are caused by large motions of domain III relative to the ChT-like domains. This was corroborated after calculating the RMSD for the backbone atoms of ChT-like domains, as very stable time profiles were obtained in this case (Supplementary Figure S6). Moreover, RMSF values for all $3 \mathrm{CL}^{\text {pro }}$ plus $\mathrm{D}$-peptide backbone atoms calculated after fitting the trajectories in respect of the backbone atoms of ChT-like domains show the large relative fluctuations of domain III (Supplementary Figure S6), which are likely to arise from the fact that $3 \mathrm{CL}^{\text {pro }}$ was simulated in the monomeric state to reduce the computational demand. The relatively loose interactions between domain III and the ChTlike domains suggest that the latter might suffice to simulate complexes with active site ligands, thus increasing the MD simulations performance. On the other hand, the RMSF profiles sharply drop for residues beyond 306, which belong to the D-tetrapeptides (see region 3 in Supplementary Figure S6). Along with the intermolecular $\mathrm{H}$-bond time profiles (Supplementary Figure S7), this result indicates that the D-tetrapeptides keep forming favorable interactions with $3 \mathrm{CL}^{\text {pro }}$ active site residues during all the simulation time.

The RMSD time profiles for 4P1 backbone atoms calculated after fitting the trajectories with respect to the backbone atoms of ChT-like domains indicate that the $\mathrm{D}$-peptide remains bound in conformations similar to that of the starting structure throughout the microsecond-long MD simulations (Supplementary Figure S8). Nonetheless, we observed several transitions between slightly different conformations in both backward and forward directions during the replicate MD simulations of this complex. The previous result was confirmed by the FEL obtained by projecting the concatenated $1 \mu$ s trajectories onto PC1 and PC2 (Figure 6). Indeed, two main local minima or basins (termed 4P1-0 and 4P1-1, the former being more populated) are observed in the FEL heatmap (Figure 6). The $3 \mathrm{CL}^{\mathrm{pro}} / 4 \mathrm{P} 1$ central structures corresponding to the main basins display slight differences in the conformation adopted by 4P1 ASN side-chain and ACE-GLY in the $\mathrm{S} 1$ and $\mathrm{S}^{\prime}$ subsites, respectively (Supplementary Figure S9). Interestingly, the 4P1-1 conformation suggests that $\mathrm{ASN}(\mathrm{OD} 1)$ and $\mathrm{ASN}(\mathrm{ND} 2)$ at $\mathrm{P} 1$ can form alternate and less prevalent $\mathrm{H}$-bonds with $\mathrm{C} 145(\mathrm{~N})$ and S144(OG), respectively (Supplementary Figure S9).

The long $\mathrm{MD}$ simulations for $3 \mathrm{CL}^{\text {pro }} / 4 \mathrm{P} 2$ and $3 \mathrm{CL}^{\text {pro }} / 4 \mathrm{P} 3$ complexes show stable peptide RMSD patterns (Supplementary Figure S8). FEL heatmaps in Figure 6 also indicate that 4P2 and 4P3 sampled conformations around a single energy basin during most of the simulation time. The central structures of both complexes corresponding to their respective minima (4P2-0 and 4P3-0) (Supplementary Figure S9) are very similar to those previously calculated from the $110 \mathrm{~ns}$ MD simulations (Figure 4). However, we found minor differences in the accommodation of 4P2-0 HIE side-chain at P1, which forms $\mathrm{H}$-bonds with $\mathrm{S} 144(\mathrm{O})$ instead of the H-bond with H163(NE2), more prevalent in the shorter $\mathrm{MD}$ simulation (Figure 4 and Supplementary Figure S9). Further calculations indicated that both H-bonds occur during the $2 \mu$ s concatenated $\mathrm{MD}$ simulations with 67 and $20 \%$ occupancies, respectively. Moreover, the 4P2-0 GLY residue at S2' interacts preferentially with T26(OG1) rather than with G143(N) and C145(N) (Figure 4 and Supplementary Figure S9). On the other hand, no appreciable differences between 4P3-0 and the central structure of $4 \mathrm{P} 3$ corresponding to the shorter MD simulation are observed (Figure 4 and Supplementary Figure S9).

Contrary to what was observed for the previously-analyzed complexes, significant variations in the peptide RMSD patterns occur along the two replicate $1 \mu \mathrm{sD}$ simulations of the $3 \mathrm{CL}^{\mathrm{pro}} /$ 4P4 complex (Supplementary Figure S8). Because of such instabilities, a third $1 \mu \mathrm{s}$ MD simulation was run for this complex, which also shows wide peptide RMSD variations (Supplementary Figure S8). In agreement with the depicted RMSD patterns, the FEL for $3 \mathrm{CL}^{\mathrm{pro}} / 4 \mathrm{P} 4$ indicates the existence of four main energy minima, termed 4P4-0 to 4P4-3, involving relatively large motions along $\mathrm{PC1}$ and $\mathrm{PC} 2$ (Figure 6). The central structures corresponding to those minima also display appreciable divergence, especially 4P4-1 and 4P4-3 with respect to 4P4-0 and 4P4-2 (Supplementary Figure S9).

In principle, the stability of the sampled minima can be estimated from their relative abundances, i.e., the fraction of trajectory frames belonging to each minimum. However, undersampling can still occur even in microsecond-long simulations, especially if transitions between different states do not occur several times in both directions during the simulation time, as in the present case (Supplementary Figure S8). This issue precludes the accurate calculation of conformational population sizes at equilibrium. Therefore, we decided to calculate the binding free energies $\left(\Delta G_{b i n d}\right)$ for the four main conformations of the $3 \mathrm{CL}^{\mathrm{pro}} /$ $4 \mathrm{P} 4$ complex sampled during the long MD simulations (see Supplementary Text $\mathbf{S 1}$ and references cited therein). Interestingly, the results show that conformations 4P4-0 and 4P4-2 have similar $\Delta G_{b i n d}$ values, considering the uncertainties of the calculated mean values $(-5.2 \pm 0.4$ and $-5.8 \pm 0.4 \mathrm{kcal} / \mathrm{mol}$, respectively, Supplementary Table S2) and the errors of $\sim 1 \mathrm{kcal} /$ mol associated with the employed technique (Aldeghi et al., 2016). Of note, inhibition constants $\left(K_{i}\right)$ ranging from 50 to $100 \mu \mathrm{M}$ are expected from the previous $\Delta G_{b i n d}$ values, in agreement with the micromolar inhibition potency displayed 

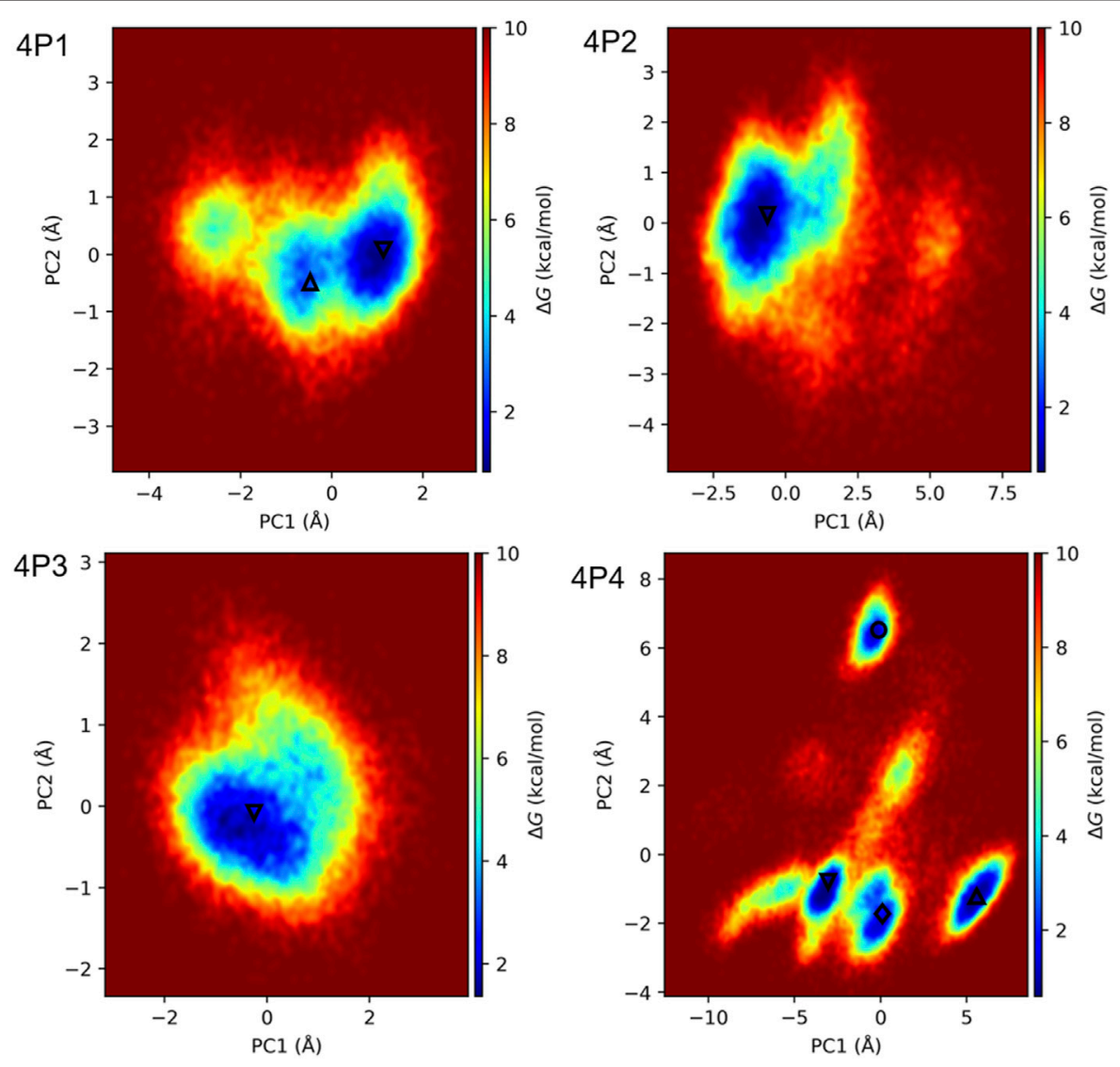

FIGURE 6|FEL heatmaps for $3 \mathrm{CL}^{\text {pro }}$ in complex with 4P1, 4P2, 4P3, and 4P4. FELs were obtained by projecting the concatenated replicate $1 \mu \mathrm{ss}$ trajectories of the complexes onto PC1 and PC2, associated with the motions of the D-peptide Ca atoms. All trajectories were fitted to the respective initial structures with respect to the backbone atoms of $3 \mathrm{CL}^{\text {pro }}$ in the ChT-like domains. The local energy minima observed in the heatmaps are indicated with the symbols $\Delta, \nabla, \diamond$, and $\mathrm{O}$, which rank the minima according to their relative sizes in decreasing order $(\Delta>\nabla>\nabla>0)$. PC1 and PC2 were chosen to generate FELS because they account for more than $68 \%$ of the motions of the D-peptide $\mathrm{C} \alpha$ atoms in all cases (Supplementary Figure S10).

by $4 \mathrm{P} 4$ (Figure 5). The other two conformations, i.e., 4P1-1 and $4 \mathrm{P} 4-3$, are significantly less stable $(-0.8 \pm 0.4$ and $-1.9 \pm 0.3 \mathrm{kcal} /$ mol, respectively, Supplementary Table S2). Overall, the free energy calculations show that the relative stabilities of the different sampled conformations do not match the results expected from the relative sizes of the four main energy minima observed in the $3 \mathrm{CL}^{\mathrm{pro}} / 4 \mathrm{P} 4$ FEL.

The two lowest-energy and nearly-isoenergetic conformations of 4P4 (4P-0 and 4P4-2, Supplementary Figure S9) differ mainly in the accommodation of HIE1 and ACE in $\mathrm{S}^{\prime}$ side of the active site. In fact, 4P4-0 HIE1 forms an H-bond with C44(O), whereas 4P4-2 HIE1 forms an H-bond with $\mathrm{H} 41(\mathrm{O})$ (Supplementary Figure S9). The remaining residues in both conformations occupy the same subsites, although some small differences in their positions are observed (Supplementary Figure S9). On the other hand, the $3 \mathrm{CL}^{\mathrm{pro}} / 4 \mathrm{P} 4$ central structure determined from the $110 \mathrm{~ns} \mathrm{MD}$ simulation (Figure 4) adopts roughly the same conformation of 4P4-0 HIE1 at $\mathrm{S}^{\prime}$, whereas the accommodation of the remaining residues in the former structure resembles to a larger extent that of 4P4-2 (Figure 4 and Supplementary Figure S9).

\section{DISCUSSION}

This work reports the in silico identification and in vitro validation of promising D-peptide inhibitors of SARS-CoV-2 $3 \mathrm{CL}^{\text {pro }}$. An in-house D-peptide library was built from scratch to search for potential $3 \mathrm{CL}^{\text {pro }}$ inhibitors through a computational workflow comprising SBVS with AutoDock Vina and several rescoring steps (Figure 1). This workflow was already employed elsewhere to predict nonpeptidic allosteric inhibitors against the malarial protease falcipain-2 (Hernandez Gonzalez et al., 2021) and was adapted here for D-peptide SBVS. To our knowledge, our study is the first to employ a docking-based approach to screen D-peptide libraries against protein targets.

Protein/peptide docking remains a challenging task due to the high flexibility of peptides, which undermines the prediction of accurate bound conformations (Rentzsch and Renard, 2015; Ciemny et al., 2018; Hashemi et al., 2021). To address this issue, existing protein/peptide docking methodologies have relied on different strategies. Peptide poses can be generated on the fly, and the most favorable ones can be selected according to their binding energy scores (Morris et al., 1998; Ewing et al., 
2001; Staneva and Wallin, 2009; Hashemi et al., 2021). However, this approach, coined as de novo docking, is not suitable for larger peptides, as exhaustive conformational sampling becomes prohibitive due to the presence of many freely-rotatable covalent bonds (Yan et al., 2017; Ansar and Vetrivel, 2019). This hurdle can be potentially overcome by conducting ensemble docking, which involves the generation of peptide conformations that can be subsequently docked into the protein binding site through rigid docking (Yan et al., 2017; Ciemny et al., 2018; Zhou et al., 2018; Ansar and Vetrivel, 2019). Moreover, MD simulationbased refinement steps can improve the accuracy of docked peptide poses (Trellet et al., 2013; Schindler et al., 2015; Yan et al., 2016; Ciemny et al., 2018; Hashemi et al., 2021).

Despite the availability of multiple peptide/protein docking tools (Ciemny et al., 2018; Hashemi et al., 2021), their efficient integration into SBVS campaigns is not straightforward (Ansar and Vetrivel, 2019). Recently, Ansar and Vetrivel presented PepVis, a pipeline for peptide SBVS following an ensemble docking approach (Ansar and Vetrivel, 2019). Their pipeline involves the generation of multiple peptide conformations that are rigidly docked into the targets and subsequently rescored and refined. As in PepVis, the D-peptide library screened in our work was created from the sequence and the starting structures were solvated and energyminimized. However, to keep things simpler, we intentionally built very small D-peptide (three and four residues) libraries, thus avoiding issues like predicting the peptide secondary structure (Ansar and Vetrivel, 2019). This was possible in our case, as we concluded by visually inspecting several crystal structures of $3 \mathrm{CL}^{\text {pro }}$ in complex with peptidomimetic inhibitors that D-tripeptides and especially D-tetrapeptides are large enough to occupy the main pockets of the enzyme's active site. Given the small size of the screened D-peptides, the ensemble approach was deemed unnecessary as it would have required additional computational steps. Instead, bound conformations during SBVS were generated and scored by the docking algorithm, as customary for small molecules. Subsequent rescoring steps in our workflow, such as generating multiple poses with AutoDock Vina using increased exhaustiveness of the search and different random seeds and MD simulations, aimed to enhance the conformational sampling of the analyzed D-peptides.

To prepare and screen libraries of larger D-peptides starting from the sequence, additional steps related to the peptide structure prediction and conformational search will be required. Existing methods and pipelines devised for protein/peptide docking (Yan et al., 2017; Ciemny et al., 2018; Ansar and Vetrivel, 2019; Hashemi et al., 2021) can be readily used if mirror images of the L-peptide structures (Garton et al., 2018) are created prior to the search for bound poses. However, the most straightforward strategy, inspired by mirror-image phage display experiments to identify D-peptide ligands (Schumacher et al., 1996), is to invert the configuration of the target protein $\mathrm{Ca}$ atoms and use an L-peptide library for SBVS.

The $\mathrm{D}$-peptides proposed here as $3 \mathrm{CL}^{\mathrm{pro}}$ inhibitors are mostly made up of aromatic residues plus HIS. Initially, we were intrigued by the fact that LEU was not found in any position of the selected D-peptides, although both SARS-CoV and SARSCoV-2 3CL ${ }^{\text {pross }}$ prefer this residue at P2 (Chuck et al., 2010; Rut et al., 2021). However, at least in multiple heterochiral peptides reported by Rut et al., the preference for LEU at P2 is restricted to the L-enantiomer (Rut et al., 2021), which might explain why LEU is absent from the identified D-peptides. Furthermore, the predicted variable accommodation of D-peptide backbones along the $3 \mathrm{CL}^{\text {pro }}$ active site, divergent from the canonical conformation of the L-peptides (Figure 3), implies that specificity profiles obtained for L-peptide substrates cannot be straightforwardly extrapolated to small D-peptides. Finally, it is worth noting that the S2 subsite of $3 \mathrm{CL}^{\text {pro }}$ can accommodate bulky aromatic moieties, e.g., 3-fluoro-L-PHE (PDB: 6M0K) (Dai et al., 2020), 4-nitro-L-phenylalanine $\left(\mathrm{PHE}\left(4-\mathrm{NO}_{2}\right)\right)$ and 2,3-dihydro-Ltryptophane (Dht) (Rut et al., 2021), thus demonstrating that there is room in this subsite for large residues like those observed in the predicted D-peptides.

Apart from the previous factors, the absence of aliphatic residues in the identified D-peptides in favor of aromatic residues may arise from a persistent bias in our workflow toward larger ligands. In fact, it is known that Autodock Vina and other docking algorithms tend to overestimate the affinity of large ligands (Chang et al., 2010). Nonetheless, as mentioned before, the impact of such bias on the final results was reduced by the subsequent rescoring steps combining MM-GBSA free energy calculations and MD simulations. Interestingly, in a previous work, two L-peptides, HHYWH and HYWWT, identified as potential $3 \mathrm{CL}^{\text {pro }}$ inhibitors using AutoDock Vina (Porto, 2021), showed a high content of HIS, TYR, and TRP, in resemblance to our results. This coincidence seems to reinforce the occurrence of a bias toward the former residues in the docking algorithm.

Despite the inaccuracies of the employed computational techniques pointed out earlier, the inhibition assays carried out for the four top-ranked D-peptides $(4 \mathrm{P} 1,4 \mathrm{P} 2,4 \mathrm{P} 3$, and $4 \mathrm{P} 4)$ validated our predictions. The tested $\mathrm{D}$-tetrapeptides displayed significant inhibition of $3 \mathrm{CL}^{\text {pro }}$ activity at $20 \mu \mathrm{M}$, causing $55-85 \%$ loss of activity in all cases. Moreover, the FELs obtained from microsecond-long MD simulations conducted for $3 \mathrm{CL}^{\text {pro }}$ bound to $4 \mathrm{P} 1,4 \mathrm{P} 2$, and $4 \mathrm{P} 3$ showed the stability of such complexes during the simulation time and that they sampled conformations around one or two similar main energy minima. Conversely, 4P4 sampled several wellseparated energy minima and relatively-large peptide RMSD variations along the replicate $1 \mu \mathrm{s} \mathrm{MD}$ trajectories. However, further energetic analyses indicated that this D-peptide coexists as two nearly-isoenergetic conformations with binding free energies consistent with its experimental inhibitory potency. In general, the main conformations of the tested D-peptides obtained from the long MD simulations were similar to those of the $110 \mathrm{~ns}$ MD simulations that were conducted as part of the presented in silico workflow. Therefore, we believe that short MD simulations are sufficient to identify promising ligands. On the other hand, longer MD simulations can be conducted after experimental validation to predict more accurate complex conformations that can be used as starting points for structure-based optimization of the hits.

The predicted structures of $3 \mathrm{CL}^{\text {pro }}$ in complex with the selected D-peptides indicate the occurrence of significant intermolecular $\mathrm{H}$-bonds present in the available crystal structures of this protease and its close homologue SARS-CoV 
$3 \mathrm{CL}^{\text {pro }}$. In this sense, we observed the formation of $\mathrm{H}$-bonds between the amide oxygen of ASN/GLN of the D-peptides and H163(NE2), which explains the strong preference for GLN at P1 (Singh et al., 2020), in several predicted complexes, including that of the tested D-peptide 4P1. Other D-peptides, such as 3P1 and $4 \mathrm{P} 2$, were found to accommodate THR and HIE at the S1 subsite and to either mimic the H-bonds formed between GLN in L-peptides and H163(NE2) or to form alternate stabilizing interactions within the subsite. Of note, D-amino acids with side-chains smaller than GLN could reach the bottom of the S1 subsite because the backbones of the analyzed D-peptides can lie closer to the pocket entrance than the L-peptide backbones. Overall, the previous results show that our workflow was able to capture the interactions underlying the fine-tuned specificity of the S1 subsite of 3CL ${ }^{\text {pro }}$ (Rut et al., 2021).

D-peptides are considered attractive therapeutic agents (Liu et al., 2016). However, this type of molecule has not been explored as potential ligands of $3 \mathrm{CL}^{\text {pro }}$ active site until now. Therefore, the tested D-tetrapeptides 4P1, 4P2, 4P3, and 4P4 expand the chemical repertoire of known $3 \mathrm{CL}^{\text {pro }}$ inhibitors that can help combat Covid19. Beyond this concrete example, the computational workflow presented here can contribute to the fast discovery of small D-peptide ligands targeting different $3 \mathrm{CL}^{\text {pro }}$ variants that can arise under viral adaptation to drug pressure (Padhi and Tripathi, 2021), as well as other proteins of interest.

\section{DATA AVAILABILITY STATEMENT}

The original contributions presented in the study are included in the article/Supplementary Material, further inquiries can be directed to the corresponding author.

\section{REFERENCES}

Abraham, M. J., Murtola, T., Schulz, R., Páll, S., Smith, J. C., Hess, B., et al. (2015). GROMACS: High Performance Molecular Simulations through Multi-Level Parallelism from Laptops to Supercomputers. SoftwareX 1-2, 19-25. doi:10.1016/j.softx.2015.06.001

Aldeghi, M., Heifetz, A., Bodkin, M. J., Knapp, S., and Biggin, P. C. (2016). Accurate Calculation of the Absolute Free Energy of Binding for Drug Molecules. Chem. Sci. 7, 207-218. doi:10.1039/c5sc02678d

Alves, V. M., Bobrowski, T., Melo-Filho, C. C., Korn, D., Auerbach, S., Schmitt, C., et al. (2021). QSAR Modeling of SARS-CoV MproInhibitors Identifies Sufugolix, Cenicriviroc, Proglumetacin, and Other Drugs as Candidates for Repurposing against SARS-CoV-2. Mol. Inf. 40, 2000113. doi:10.1002/ minf.202000113

Amadei, A., Linssen, A. B. M., and Berendsen, H. J. C. (1993). Essential Dynamics of Proteins. Proteins 17, 412-425. doi:10.1002/prot.340170408

Amin, S. A., Banerjee, S., Ghosh, K., Gayen, S., and Jha, T. (2021). Protease Targeted COVID-19 Drug Discovery and its Challenges: Insight into Viral Main Protease (Mpro) and Papain-like Protease (PLpro) Inhibitors. Bioorg. Med. Chem. 29, 115860. doi:10.1016/j.bmc.2020.115860

Ansar, S., and Vetrivel, U. (2019). PepVis: An Integrated Peptide Virtual Screening Pipeline for Ensemble and Flexible Docking Protocols. Chem. Biol. Drug Des. 94, 2041-2050. doi:10.1111/cbdd.13607

Banerjee, S., Yadav, S., Banerjee, S., Fakayode, S. O., Parvathareddy, J., Reichard, W., et al. (2021). Drug Repurposing to Identify Nilotinib as a

\section{AUTHOR CONTRIBUTIONS}

JEHG, RJE, MAC Methodology, Investigation, Validation, Formal analysis. MAC Conceptualisation. JEHG Writing. DW Resources and Editing. RJE, MAC Reviewing and Editing.

\section{FUNDING}

This work was supported in part by São Paulo Research Foundation (FAPESP), Grant 2020/10214-1, and Coordenação de Aperfeiçoamento de Pessoal de Nível Superior (CAPES), Edital 09/2020 Pandemias, Grant 88881.503808/2020-01.

\section{ACKNOWLEDGMENTS}

We would like to thank the support of the Institute of Biological Information Processing (IBI-7) Forschungszentrum Jülich, Germany and of the National Laboratory for Scientific Computing (LNCC/MCTI, Brazil) for providing HPC resources of the SDumont supercomputer, which have contributed to the research results reported within this work (http://sdumont.lncc.br). We are grateful to our Brazilian partner, whom the federal government is constantly neglecting.

\section{SUPPLEMENTARY MATERIAL}

The Supplementary Material for this article can be found online at: https:/www.frontiersin.org/articles/10.3389/fmolb.2021.816166/ full\#supplementary-material

Potential SARS-CoV-2 Main Protease Inhibitor: Insights from a Computational and In Vitro Study. J. Chem. Inf. Model. 61, 5469-5483. doi:10.1021/acs.jcim.1c00524

Berendsen, H. J. C., Postma, J. P. M., van Gunsteren, W. F., DiNola, A., and Haak, J. R. (1984). Molecular Dynamics with Coupling to an External bath. J. Chem. Phys. 81, 3684-3690. doi:10.1063/1.448118

Case, D. A., Belfon, K., Ben-Shalom, I. Y., Brozell, S. R., Cerutti, D. S., Cheatham, T. E., III, et al. (2020). Amber 2020. San Francisco: University of California.

Chang, M. W., Ayeni, C., Breuer, S., and Torbett, B. E. (2010). Virtual Screening for HIV Protease Inhibitors: a Comparison of AutoDock 4 and Vina. PLoS One 5, e11955. doi:10.1371/journal.pone.0011955

Chen, R., and Weng, Z. (2002). Docking Unbound Proteins Using Shape Complementarity, Desolvation, and Electrostatics. Proteins 47, 281-294. doi: $10.1002 /$ prot. 10092

Chia, C. S. B., Xu, W., and Shuyi Ng, P. (2021). A Patent Review on SARS Coronavirus Main Protease $\left(3 \mathrm{CL}^{\mathrm{pro}}\right)$ Inhibitors. Chem. Med. Chem. doi:10.1002/cmdc.202100576

Chowdhury, K. H., Chowdhury, M. R., Mahmud, S., Tareq, A. M., Hanif, N. B., Banu, N., et al. (2020). Drug Repurposing Approach against Novel Coronavirus Disease (COVID-19) through Virtual Screening Targeting SARS-CoV-2 Main Protease. Biology 10, 2. doi:10.3390/biology10010002

Chuck, C.-P., Chong, L.-T., Chen, C., Chow, H.-F., Wan, D. C.-C., and Wong, K.-B. (2010). Profiling of Substrate Specificity of SARS-CoV 3CLpro. PLoS One 5, e13197. doi:10.1371/journal.pone.0013197

Ciemny, M., Kurcinski, M., Kamel, K., Kolinski, A., Alam, N., Schueler-Furman, O., et al. (2018). Protein-peptide Docking: Opportunities and Challenges. Drug 
Discov. Today 23, 1530-1537. doi:10.1016/j.drudis.2018.05.006

Connolly, M. L. (1983). Analytical Molecular Surface Calculation. J. Appl. Cryst. 16, 548-558. doi:10.1107/S0021889883010985

Dai, W., Zhang, B., Jiang, X.-M., Su, H., Li, J., Zhao, Y., et al. (2020). Structurebased Design of Antiviral Drug Candidates Targeting the SARS-CoV-2 Main Protease. Science 368, 1331-1335. doi:10.1126/science.abb4489

Darden, T., York, D., and Pedersen, L. (1993). Particle Mesh Ewald: AnN.Log(N) Method for Ewald Sums in Large Systems. J. Chem. Phys. 98, 10089-10092. doi:10.1063/1.464397

DeLano, W. L. (2002). PyMOL. San Carlos, CA: PyMOL, 700.

Dolinsky, T. J., Czodrowski, P., Li, H., Nielsen, J. E., Jensen, J. H., Klebe, G., et al. (2007). PDB2PQR: Expanding and Upgrading Automated Preparation of Biomolecular Structures for Molecular Simulations. Nucleic Acids Res. 35, W522-W525. doi:10.1093/nar/gkm276

Douangamath, A., Fearon, D., Gehrtz, P., Krojer, T., Lukacik, P., Owen, C. D., et al. (2020). Crystallographic and Electrophilic Fragment Screening of the SARS-CoV2 Main Protease. Nat. Commun. 11, 5047. doi:10.1038/s41467-020-18709-w

Eberle, R. J., Olivier, D. S., Amaral, M. S., Gering, I., Willbold, D., Arni, R. K., et al. (2021). The Repurposed Drugs Suramin and Quinacrine Cooperatively Inhibit SARS-CoV-2 3CLpro In Vitro. Viruses 13, 873. doi:10.3390/v13050873

Ewing, T. J. A., Makino, S., Skillman, A. G., and Kuntz, I. D. (2001). DOCK 4.0: Search Strategies for Automated Molecular Docking of Flexible Molecule Databases. J. Comput. Aided Mol. Des. 15, 411-428. doi:10.1023/a: 1011115820450

Federico, L. B., Silva, G. M., da Silva Hage-Melim, L. I., Gomes, S. Q., Barcelos, M. P., Galindo Francischini, I. A., et al. (2021). Identification of Known Drugs as Potential SARS-CoV-2 Mpro Inhibitors Using Ligand- and Structure-Based Virtual Screening. Future Med. Chem. 13, 1353-1366. doi:10.4155/fmc-20210025

Funke, S. A., and Willbold, D. (2009). Mirror Image Phage Display-A Method to Generate D-Peptide Ligands for Use in Diagnostic or Therapeutical Applications. Mol. Biosyst. 5, 783-786. doi:10.1039/b904138a

Garton, M., Nim, S., Stone, T. A., Wang, K. E., Deber, C. M., and Kim, P. M. (2018). Method to Generate Highly Stable D-Amino Acid Analogs of Bioactive Helical Peptides Using a Mirror Image of the Entire PDB. Proc. Natl. Acad. Sci. USA 115, 1505-1510. doi:10.1073/pnas.1711837115

Ghahremanpour, M. M., Tirado-Rives, J., Deshmukh, M., Ippolito, J. A., Zhang, C.H., Cabeza de Vaca, I., et al. (2020). Identification of 14 Known Drugs as Inhibitors of the Main Protease of SARS-CoV-2. ACS Med. Chem. Lett. 11, 2526-2533. doi:10.1021/acsmedchemlett.0c00521

Gogoi, M., Borkotoky, M., Borchetia, S., Chowdhury, P., Mahanta, S., and Barooah, A. K. (2021). Black tea Bioactives as Inhibitors of Multiple Targets of SARSCoV-2 (3CLpro, PLpro and RdRp): a Virtual Screening and Molecular Dynamic Simulation Study. J. Biomol. Struct. Dyn. 1, 24. doi:10.1080/ 07391102.2021.1897679

Gohlke, H., and Case, D. A. (2004). Converging Free Energy Estimates: MM$\mathrm{PB}(\mathrm{GB}) \mathrm{SA}$ Studies on the Protein-Protein Complex Ras-Raf. J. Comput. Chem. 25, 238-250. doi:10.1002/jcc.10379

Gohlke, H., Kiel, C., and Case, D. A. (2003). Insights into Protein-Protein Binding by Binding Free Energy Calculation and Free Energy Decomposition for the Ras-Raf and Ras-RalGDS Complexes. J. Mol. Biol. 330, 891-913. doi:10.1016/ s0022-2836(03)00610-7

Gorbalenya, A. E., Koonin, E. V., Donchenko, A. P., and Blinov, V. M. (1989). Coronavirus Genome: Prediction of Putative Functional Domains in the Nonstructural Polyprotein by Comparative Amino Acid Sequence Analysis. Nucl. Acids Res. 17, 4847-4861. doi:10.1093/nar/17.12.4847

GraphPad (2018). One-way ANOVA Followed by Dunnett's Multiple Comparisons Test Was Performed Using GraphPad Prism Version 8.0.0 for Windows. San Diego, California, USA: GraphPad. Available at: www.graphpad.com.

Guedes, I. A., Costa, L. S. C., Dos Santos, K. B., Karl, A. L. M., Rocha, G. K., Teixeira, I. M., et al. (2021). Drug Design and Repurposing with DockThor-VS Web Server Focusing on SARS-CoV-2 Therapeutic Targets and Their Nonsynonym Variants. Sci. Rep. 11, 5543. doi:10.1038/s41598-021-84700-0

Günther, S., Reinke, P. Y. A., Fernández-García, Y., Lieske, J., Lane, T. J., Ginn, H. M., et al. (2021). X-ray Screening Identifies Active Site and Allosteric Inhibitors of SARS-CoV-2 Main Protease. Science 372, 642-646. doi:10.1126/ science.abf7945
Guo, S., Xie, H., Lei, Y., Liu, B., Zhang, L., Xu, Y., et al. (2021). Discovery of Novel Inhibitors against Main Protease (Mpro) of SARS-CoV-2 via Virtual Screening and Biochemical Evaluation. Bioorg. Chem. 110, 104767. doi:10.1016/ j.bioorg.2021.104767

Gupta, A., Rani, C., Pant, P., Vijayan, V., Vikram, N., Kaur, P., et al. (2020). Structure-Based Virtual Screening and Biochemical Validation to Discover a Potential Inhibitor of the SARS-CoV-2 Main Protease. ACS Omega 5, 33151-33161. doi:10.1021/acsomega.0c04808

Gupta, Y., Maciorowski, D., Zak, S. E., Jones, K. A., Kathayat, R. S., Azizi, S.-A., et al. (2021). Bisindolylmaleimide IX: A Novel Anti-SARS-CoV2 Agent Targeting Viral Main Protease 3CLpro Demonstrated by Virtual Screening Pipeline and In-Vitro Validation Assays. Methods 195, 57-71. doi:10.1016/j.ymeth.2021.01.003

Hamdy, R., Fayed, B., Mostafa, A., Shama, N. M. A., Mahmoud, S. H., Mehta, C. H., et al. (2021). Iterated Virtual Screening-Assisted Antiviral and Enzyme Inhibition Assays Reveal the Discovery of Novel Promising Anti-SARS-CoV-2 with Dual Activity. Int. J. Mol. Sci. 22, 9057. doi:10.3390/ijms22169057

Hashemi, Z. S., Zarei, M., Fath, M. K., Ganji, M., Farahani, M. S., Afsharnouri, F., et al. (2021). In Silico Approaches for the Design and Optimization of Interfering Peptides against Protein-Protein Interactions. Front. Mol. Biosci. 8, 669431. doi:10.3389/fmolb.2021.669431

Hegyi, A., and Ziebuhr, J. (2002). Conservation of Substrate Specificities Among Coronavirus Main Proteases. J. Gen. Virol. 83, 595-599. doi:10.1099/00221317-83-3-595

Hernández González, J. E., Hernández Alvarez, L., Pascutti, P. G., and Valiente, P. A. (2017). Predicting Binding Modes of Reversible Peptide-Based Inhibitors of Falcipain-2 Consistent with Structure-Activity Relationships. Proteins 85, 1666-1683. doi:10.1002/prot.25322

Hernández González, J. E., Salas-Sarduy, E., Hernández Alvarez, L., Barreto Gomes, D. E., Pascutti, P. G., Oostenbrink, C., et al. (2021). In Silico identification of Noncompetitive Inhibitors Targeting an Uncharacterized Allosteric Site of Falcipain-2. J. Comput. Aided Mol. Des. 35, 1067-1079. doi:10.1007/s10822-021-00420-7

Hess, B. (2002). Determining the Shear Viscosity of Model Liquids from Molecular Dynamics Simulations. J. Chem. Phys. 116, 209-217. doi:10.1063/1.1421362

Hou, T., Wang, J., Li, Y., and Wang, W. (2011). Assessing the Performance of the Molecular mechanics/Poisson Boltzmann Surface Area and Molecular Mechanics/generalized Born Surface Area Methods. II. The Accuracy of Ranking Poses Generated from Docking. J. Comput. Chem. 32, 866-877. doi:10.1002/jcc.21666

Jin, Z., Du, X., Xu, Y., Deng, Y., Liu, M., Zhao, Y., et al. (2020). Structure of Mpro from SARS-CoV-2 and Discovery of its Inhibitors. Nature 582, 289-293. doi:10.1038/s41586-020-2223-y

Jukič, M., Janežič, D., and Bren, U. (2020). Ensemble Docking Coupled to Linear Interaction Energy Calculations for Identification of Coronavirus Main Protease (3CLpro) Non-covalent Small-Molecule Inhibitors. Molecules 25, 5808. doi:10.3390/molecules25245808

Kiemer, L., Lund, O., Brunak, S., and Blom, N. (2004). Coronavirus 3CLpro Proteinase Cleavage Sites: Possible Relevance to SARS Virus Pathology. BMC Bioinformatics 5, 72. doi:10.1186/1471-2105-5-72

Kneller, D. W., Phillips, G., O’Neill, H. M., Jedrzejczak, R., Stols, L., Langan, P., et al. (2020). Structural Plasticity of SARS-CoV-2 3CL Mpro Active Site Cavity Revealed by Room Temperature X-ray Crystallography. Nat. Commun. 11, 3202. doi:10.1038/s41467-020-16954-7

Kumar, A., Mishra, D. C., Angadi, U. B., Yadav, R., Rai, A., and Kumar, D. (2021). Inhibition Potencies of Phytochemicals Derived from Sesame against SARSCoV-2 Main Protease: A Molecular Docking and Simulation Study. Front. Chem. 9, 744376. doi:10.3389/fchem.2021.744376

Li, P., Song, L. F., and Merz, K. M., Jr. (2015). Systematic Parameterization of Monovalent Ions Employing the Nonbonded Model. J. Chem. Theor. Comput. 11, 1645-1657. doi:10.1021/ct500918t

Li, Z., Li, X., Huang, Y.-Y., Wu, Y., Liu, R., Zhou, L., et al. (2020). Identify Potent SARS-CoV-2 Main Protease Inhibitors via Accelerated Free Energy Perturbation-Based Virtual Screening of Existing Drugs. Proc. Natl. Acad. Sci. USA 117, 27381-27387. doi:10.1073/pnas.2010470117

Liu, M., Li, X., Xie, Z., Xie, C., Zhan, C., Hu, X., et al. (2016). D-peptides as Recognition Molecules and Therapeutic Agents. Chem. Rec. 16, 1772-1786. doi:10.1002/tcr.201600005 
Lokhande, K. B., Doiphode, S., Vyas, R., and Swamy, K. V. (2021). Molecular Docking and Simulation Studies on SARS-CoV-2 Mpro Reveals Mitoxantrone, Leucovorin, Birinapant, and Dynasore as Potent Drugs against COVID-19. J. Biomol. Struct. Dyn. 39, 7294-7305. doi:10.1080/07391102.2020.1805019

Ma, C., Sacco, M. D., Hurst, B., Townsend, J. A., Hu, Y., Szeto, T., et al. (2020). Boceprevir, GC-376, and Calpain Inhibitors II, XII Inhibit SARS-CoV-2 Viral Replication by Targeting the Viral Main Protease. Cell Res 30, 678-692. doi:10.1038/s41422-020-0356-z

Maier, J. A., Martinez, C., Kasavajhala, K., Wickstrom, L., Hauser, K. E., and Simmerling, C. (2015). ff14SB: Improving the Accuracy of Protein Side Chain and Backbone Parameters from ff99SB. J. Chem. Theor. Comput. 11, 3696-3713. doi:10.1021/acs.jctc.5b00255

Marra, M. A., Jones, S. J. M., Astell, C. R., Holt, R. A., Brooks-Wilson, A., Butterfield, Y. S. N., et al. (2003). The Genome Sequence of the SARSAssociated Coronavirus. Science 300, 1399-1404. doi:10.1126/science.1085953

Mengist, H. M., Dilnessa, T., and Jin, T. (2021). Structural Basis of Potential Inhibitors Targeting SARS-CoV-2 Main Protease. Front. Chem. 9, 622898. doi:10.3389/fchem.2021.622898

Meyer-Almes, F.-J. (2020). Repurposing Approved Drugs as Potential Inhibitors of 3CL-Protease of SARS-CoV-2: Virtual Screening and Structure Based Drug Design. Comput. Biol. Chem. 88, 107351. doi:10.1016/ j.compbiolchem.2020.107351

Miller, B. R., 3rd, McGee, T. D., Jr., Swails, J. M., Homeyer, N., Gohlke, H., and Roitberg, A. E. (2012). MMPBSA.py: An Efficient Program for End-State Free Energy Calculations. J. Chem. Theor. Comput. 8, 3314-3321. doi:10.1021/ ct300418h

Miyamoto, S., and Kollman, P. A. (1992). Settle: An Analytical Version of the SHAKE and RATTLE Algorithm for Rigid Water Models. J. Comput. Chem. 13, 952-962. doi:10.1002/jcc.540130805

Morris, G. M., Goodsell, D. S., Halliday, R. S., Huey, R., Hart, W. E., Belew, R. K., et al. (1998). Automated Docking Using a Lamarckian Genetic Algorithm and an Empirical Binding Free Energy Function. J. Comput. Chem. 19, 1639-1662. doi:10.1002/(SICI)1096-987X(19981115)19:14<1639:AIDJCC10>3.0.CO;2-B

Morris, G. M., Huey, R., Lindstrom, W., Sanner, M. F., Belew, R. K., Goodsell, D. S., et al. (2009). AutoDock4 and AutoDockTools4: Automated Docking with Selective Receptor Flexibility. J. Comput. Chem. 30, 2785-2791. doi:10.1002/ jcc. 21256

Muramatsu, T., Takemoto, C., Kim, Y.-T., Wang, H., Nishii, W., Terada, T., et al. (2016). SARS-CoV 3CL Protease Cleaves its C-Terminal Autoprocessing Site by Novel Subsite Cooperativity. Proc. Natl. Acad. Sci. USA 113, 12997-13002. doi:10.1073/pnas.1601327113

Naik, S. R., Bharadwaj, P., Dingelstad, N., Kalyaanamoorthy, S., Mandal, S. C., Ganesan, A., et al. (2021). Structure-based Virtual Screening, Molecular Dynamics and Binding Affinity Calculations of Some Potential Phytocompounds against SARS-CoV-2. J. Biomol. Struct. Dyn. 1, 18. doi:10.1080/07391102.2021.1891969

Nguyen, H., Roe, D. R., and Simmerling, C. (2013). Improved Generalized Born Solvent Model Parameters for Protein Simulations. J. Chem. Theor. Comput. 9, 2020-2034. doi:10.1021/ct3010485

Olubiyi, O. O., Olagunju, M., Keutmann, M., Loschwitz, J., and Strodel, B. (2020). High Throughput Virtual Screening to Discover Inhibitors of the Main Protease of the Coronavirus SARS-CoV-2. Molecules 25, 3193. doi:10.3390/ molecules 25143193

Owen, D. R., Allerton, C. M. N., Anderson, A. S., Aschenbrenner, L., Avery, M., Berritt, S., et al. (2021). An Oral SARS-CoV-2 M Pro Inhibitor Clinical Candidate for the Treatment of COVID-19. Science 374, 1586-1593. doi:10.1126/science.abl4784

Padhi, A. K., and Tripathi, T. (2021). Targeted Design of Drug Binding Sites in the Main Protease of SARS-CoV-2 Reveals Potential Signatures of Adaptation. Biochem. Biophysical Res. Commun. 555, 147-153. doi:10.1016/ j.bbrc.2021.03.118

Pan, Y., Huang, N., Cho, S., and MacKerell, A. D., Jr. (2003). Consideration of Molecular Weight during Compound Selection in Virtual Target-Based Database Screening. J. Chem. Inf. Comput. Sci. 43, 267-272. doi:10.1021/ ci020055f

Papaleo, E., Mereghetti, P., Fantucci, P., Grandori, R., and De Gioia, L. (2009). Freeenergy Landscape, Principal Component Analysis, and Structural Clustering to
Identify Representative Conformations from Molecular Dynamics Simulations: the Myoglobin Case. J. Mol. Graphics Model. 27, 889-899. doi:10.1016/ j.jmgm.2009.01.006

Pathak, N., Chen, Y.-T., Hsu, Y.-C., Hsu, N.-Y., Kuo, C.-J., Tsai, H. P., et al. (2021). Uncovering Flexible Active Site Conformations of SARS-CoV-2 3CL Proteases through Protease Pharmacophore Clusters and COVID-19 Drug Repurposing. ACS Nano 15, 857-872. doi:10.1021/acsnano.0c07383

Payne, S. (2017). Family Coronaviridae. Viruses 2017, 149-158. doi:10.1016/B9780-12-803109-4.00017-9

Porto, W. F. (2021). Virtual Screening of Peptides with High Affinity for SARSCoV-2 Main Protease. Comput. Biol. Med. 133, 104363. doi:10.1016/ j.compbiomed.2021.104363

Price, D. J., and Brooks, C. L., 3rd (2004). A Modified TIP3P Water Potential for Simulation with Ewald Summation. J. Chem. Phys. 121, 10096-10103. doi:10.1063/1.1808117

Rajpoot, S., Alagumuthu, M., and Baig, M. S. (2021). Dual Targeting of 3CLpro and PLpro of SARS-CoV-2: A Novel Structure-Based Design Approach to Treat COVID-19. Curr. Res. Struct. Biol. 3, 9-18. doi:10.1016/j.crstbi.2020.12.001

Rehman, M. T., AlAjmi, M. F., and Hussain, A. (2021). Natural Compounds as Inhibitors of SARS-CoV-2 Main Protease (3CLpro): A Molecular Docking and Simulation Approach to Combat COVID-19. Cpd 27, 3577-3589. doi:10.2174/ 1381612826999201116195851

Rentzsch, R., and Renard, B. Y. (2015). Docking Small Peptides Remains a Great challenge: an Assessment Using AutoDock Vina. Brief. Bioinform. 16, 1045-1056. doi:10.1093/bib/bbv008

Rota, P. A., Oberste, M. S., Monroe, S. S., Nix, W. A., Campagnoli, R., Icenogle, J. P., et al. (2003). Characterization of a Novel Coronavirus Associated with Severe Acute Respiratory Syndrome. Science 300, 1394-1399. doi:10.1126/ science. 1085952

Rut, W., Groborz, K., Zhang, L., Sun, X., Zmudzinski, M., Pawlik, B., et al. (2021). SARS-CoV-2 Mpro Inhibitors and Activity-Based Probes for Patient-Sample Imaging. Nat. Chem. Biol. 17, 222-228. doi:10.1038/s41589-020-00689-Z

Ryckaert, J.-P., Ciccotti, G., and Berendsen, H. J. C. (1977). Numerical Integration of the Cartesian Equations of Motion of a System with Constraints: Molecular Dynamics of N-Alkanes. J. Comput. Phys. 23, 327-341. doi:10.1016/00219991(77)90098-5

Sabbah, D. A., Hajjo, R., Bardaweel, S. K., and Zhong, H. A. (2021). An Updated Review on SARS-CoV-2 Main Proteinase (MPro): Protein Structure and SmallMolecule Inhibitors. Curr. Top. Med. Chem. 21, 442-460. doi:10.2174/ 1568026620666201207095117

Salomon-Ferrer, R., Götz, A. W., Poole, D., Le Grand, S., and Walker, R. C. (2013). Routine Microsecond Molecular Dynamics Simulations with AMBER on GPUs. 2. Explicit Solvent Particle Mesh Ewald. J. Chem. Theor. Comput. 9, 3878-3888. doi:10.1021/ct400314y

Schindler, C. E. M., de Vries, S. J., and Zacharias, M. (2015). Fully Blind PeptideProtein Docking with pepATTRACT. Structure 23, 1507-1515. doi:10.1016/ j.str.2015.05.021

Schneider, T., and Stoll, E. (1978). Molecular-dynamics Study of a ThreeDimensional One-Component Model for Distortive Phase Transitions. Phys. Rev. B 17, 1302-1322. doi:10.1103/PhysRevB.17.1302

Schumacher, T. N. M., Mayr, L. M., Minor, D. L., Jr., Milhollen, M. A., Burgess, M. W., and Kim, P. S. (1996). Identification of D -Peptide Ligands through MirrorImage Phage Display. Science 271, 1854-1857. doi:10.1126/ science.271.5257.1854

Seeliger, D., and de Groot, B. L. (2010). Ligand Docking and Binding Site Analysis with PyMOL and Autodock/Vina. J. Comput. Aided Mol. Des. 24, 417-422. doi:10.1007/s10822-010-9352-6

Selvaraj, C., Panwar, U., Dinesh, D. C., Boura, E., Singh, P., Dubey, V. K., et al. (2020). Microsecond MD Simulation and Multiple-Conformation Virtual Screening to Identify Potential Anti-COVID-19 Inhibitors against SARSCoV-2 Main Protease. Front. Chem. 8, 595273. doi:10.3389/fchem.2020.595273

Shao, J., Tanner, S. W., Thompson, N., and Cheatham, T. E. (2007). Clustering Molecular Dynamics Trajectories: 1. Characterizing the Performance of Different Clustering Algorithms. J. Chem. Theor. Comput. 3, 2312-2334. doi:10.1021/ct700119m

Singh, E., Khan, R. J., Jha, R. K., Amera, G. M., Jain, M., Singh, R. P., et al. (2020). A Comprehensive Review on Promising Anti-viral Therapeutic Candidates Identified against Main Protease from SARS-CoV-2 through Various 
Computational Methods. J. Genet. Eng. Biotechnol. 18, 69. doi:10.1186/s43141020-00085-z

Sisakht, M., Mahmoodzadeh, A., and Darabian, M. (2021). Plant-derived Chemicals as Potential Inhibitors of SARS-CoV -2 Main Protease ( 6LU7 ), a Virtual Screening Study. Phytotherapy Res. 35, 3262-3274. doi:10.1002/ ptr.7041

Staneva, I., and Wallin, S. (2009). All-atom Monte Carlo Approach to ProteinPeptide Binding. J. Mol. Biol. 393, 1118-1128. doi:10.1016/ j.jmb.2009.08.063

Sun, N., Funke, S. A., and Willbold, D. (2012). Mirror Image Phage Display Generating Stable Therapeutically and Diagnostically Active Peptides with Biotechnological Means. J. Biotechnol. 161, 121-125. doi:10.1016/ j.jbiotec.2012.05.019

Tahir Ul Qamar, M., Alqahtani, S. M., Alamri, M. A., and Chen, L.-L. (2020). Structural Basis of SARS-CoV-2 3CLpro and Anti-COVID-19 Drug Discovery from Medicinal Plants. J. Pharm. Anal. 10, 313-319. doi:10.1016/ j.jpha.2020.03.009

Trellet, M., Melquiond, A. S. J., and Bonvin, A. M. J. J. (2013). A Unified Conformational Selection and Induced Fit Approach to Protein-Peptide Docking. PLoS One 8, e58769. doi:10.1371/journal.pone.0058769

Trott, O., and Olson, A. J. (2009). AutoDock Vina: Improving the Speed and Accuracy of Docking with a New Scoring Function, Efficient Optimization, and Multithreading. J. Comput. Chem. 31, 21334. doi:10.1002/jcc.21334

Valiente, P. A., Wen, H., Nim, S., Lee, J., Kim, H. J., Kim, J., et al. (2021). Computational Design of Potent D-Peptide Inhibitors of SARS-CoV-2. J. Med. Chem. 64, 14955-14967. doi:10.1021/acs.jmedchem.1c00655

Wang, C., Horby, P. W., Hayden, F. G., and Gao, G. F. (2020). A Novel Coronavirus Outbreak of Global Health Concern. The Lancet 395, 470-473. doi:10.1016/ S0140-6736(20)30185-9

Weiser, J. r., Shenkin, P. S., and Still, W. C. (1999). Approximate Atomic Surfaces from Linear Combinations of Pairwise Overlaps (LCPO). J. Comput. Chem. 20, 217-230. JCC4>3.0.CO;2-A

Wiesehan, K., and Willbold, D. (2003). Mirror-image Phage Display: Aiming at the Mirror. Chembiochem 4, 811-815. doi:10.1002/cbic.200300570

World Health Organization (2021). Coronavirus Disease (COVID-19) Weekly Epidemiological Update and Weekly Operational Update. Available at: https:// www.who.int/emergencies/diseases/novel-coronavirus-2019/situation-reports (Accessed December 18th, 2021).

Wu, C., Liu, Y., Yang, Y., Zhang, P., Zhong, W., Wang, Y., et al. (2020a). Analysis of Therapeutic Targets for SARS-CoV-2 and Discovery of Potential Drugs by Computational Methods. Acta Pharmaceutica Sinica B 10, 766-788. doi:10.1016/j.apsb.2020.02.008

Wu, F., Zhao, S., Yu, B., Chen, Y.-M., Wang, W., Song, Z.-G., et al. (2020b). A New Coronavirus Associated with Human Respiratory Disease in China. Nature 579, 265-269. doi:10.1038/s41586-020-2008-3
Yan, C., Xu, X., and Zou, X. (2016). Fully Blind Docking at the Atomic Level for Protein-Peptide Complex Structure Prediction. Structure 24, 1842-1853. doi:10.1016/j.str.2016.07.021

Yan, F., and Gao, F. (2021). An Overview of Potential Inhibitors Targeting Nonstructural Proteins 3 (PLpro and Mac1) and 5 (3CLpro/Mpro) of SARS-CoV-2. Comput. Struct. Biotechnol. J. 19, 4868-4883. doi:10.1016/j.csbj.2021.08.036

Yan, S., and Wu, G. (2021). Potential 3-chymotrypsin-like Cysteine Protease Cleavage Sites in the Coronavirus Polyproteins Ppla and Pplab and Their Possible Relevance to COVID-19 Vaccine and Drug Development. FASEB j. 35, e21573. doi:10.1096/fj.202100280RR

Yan, Y., Zhang, D., and Huang, S.-Y. (2017). Efficient Conformational Ensemble Generation of Protein-Bound Peptides. J. Cheminform 9, 59. doi:10.1186/ s13321-017-0246-7

Yang, J., Lin, X., Xing, N., Zhang, Z., Zhang, H., Wu, H., et al. (2021). StructureBased Discovery of Novel Nonpeptide Inhibitors Targeting SARS-CoV-2 Mpro. J. Chem. Inf. Model. 61, 3917-3926. doi:10.1021/acs.jcim.1c00355

Zhang, L., Lin, D., Kusov, Y., Nian, Y., Ma, Q., Wang, J., et al. (2020a). aKetoamides as Broad-Spectrum Inhibitors of Coronavirus and Enterovirus Replication: Structure-Based Design, Synthesis, and Activity Assessment. J. Med. Chem. 63, 4562-4578. doi:10.1021/acs.jmedchem.9b01828

Zhang, L., Lin, D., Sun, X., Curth, U., Drosten, C., Sauerhering, L., et al. (2020b). Crystal Structure of SARS-CoV-2 Main Protease Provides a Basis for Design of Improved a-ketoamide Inhibitors. Science 368, 409-412. doi:10.1126/science.abb3405

Zhou, P., Jin, B., Li, H., and Huang, S.-Y. (2018). HPEPDOCK: a Web Server for Blind Peptide-Protein Docking Based on a Hierarchical Algorithm. Nucleic Acids Res. 46, W443-W450. doi:10.1093/nar/gky357

Ziebuhr, J. (2005). The Coronavirus Replicase. Curr. Top. Microbiol. Immunol. 287, 57-94. doi:10.1007/3-540-26765-4_3

Conflict of Interest: The authors declare that the research was conducted in the absence of any commercial or financial relationships that could be construed as a potential conflict of interest.

Publisher's Note: All claims expressed in this article are solely those of the authors and do not necessarily represent those of their affiliated organizations, or those of the publisher, the editors and the reviewers. Any product that may be evaluated in this article, or claim that may be made by its manufacturer, is not guaranteed or endorsed by the publisher.

Copyright $\odot 2022$ Hernández González, Eberle, Willbold and Coronado. This is an open-access article distributed under the terms of the Creative Commons Attribution License (CC BY). The use, distribution or reproduction in other forums is permitted, provided the original author(s) and the copyright owner(s) are credited and that the original publication in this journal is cited, in accordance with accepted academic practice. No use, distribution or reproduction is permitted which does not comply with these terms. 